\title{
Evaluation etablierter Femurachsen zur Bestimmung der femoralen Rotation in der Kniegelenksendoprothetik unter Berücksichtigung des posterioren Femur-Offsets
}

\author{
INAUGURAL-DISSERTATION \\ zur Erlangung des Doktorgrades \\ der Medizinischen Fakultät der \\ Georg-August-Universität zu Göttingen \\ vorgelegt von \\ Tobias Franz Brodkorb \\ aus \\ Heidelberg
}

Göttingen 2017 
Dekan:

Referent:

Ko-Referent/-in:

Drittreferent/-in:
Prof. Dr. rer. nat. H. K. Kroemer

\author{
PD Dr. med. T. Walde
}

Prof. Dr. René Müller-Wille

Prof. Dr. Thomas Meyer 


\section{Inhaltsverzeichnis}

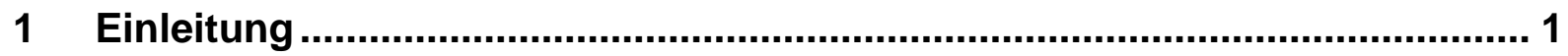

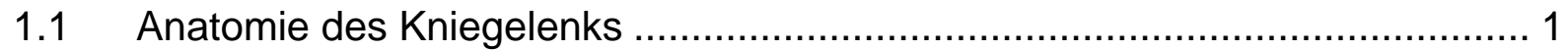

1.1.1 Knöcherne Strukturen des Kniegelenks ....................................... 1

1.1.2 Ligamentäre Strukturen des Kniegelenks .................................... 2

1.1.3 Genetische Entwicklung des Kniegelenks ................................... 4

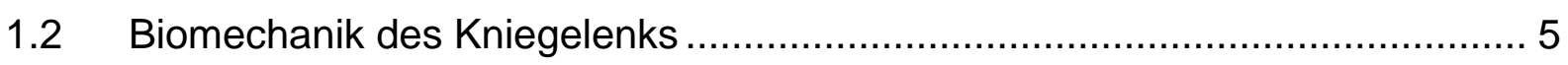

1.2.1 Beinachse und Kniebewegung .................................................. 5

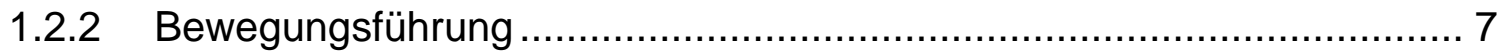

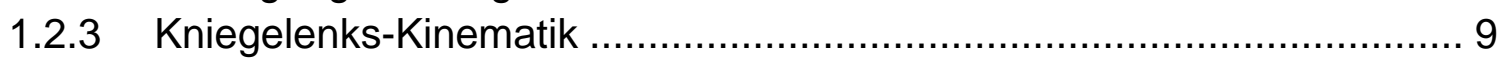

1.3 Besonderheiten des distalen Femurs mit Bezug zur Kniegelenksendoprothetik

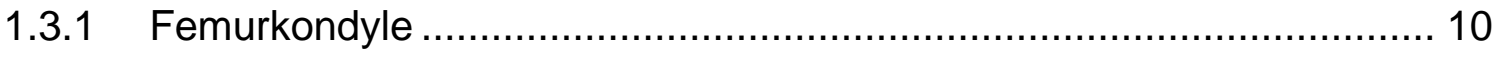

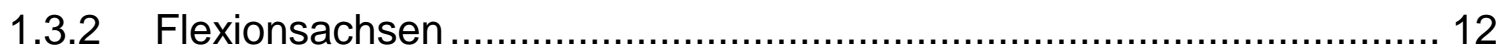

1.3.2.1 Die funktionelle Flexions- / Extensionsachse ......................... 12

1.3.2.2 Transepikondyläre Achsen und posteriore Kondyle Achse ........... 13

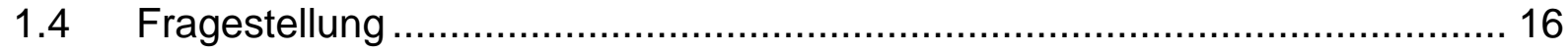

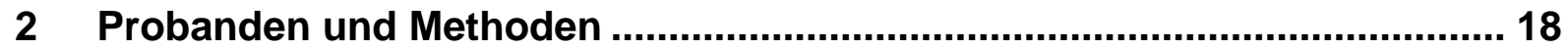

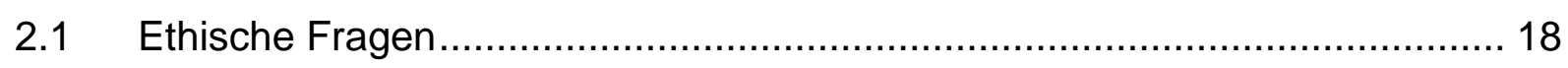

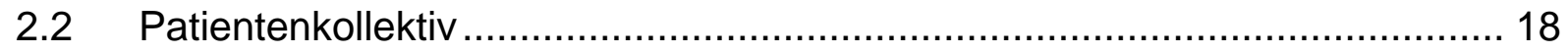

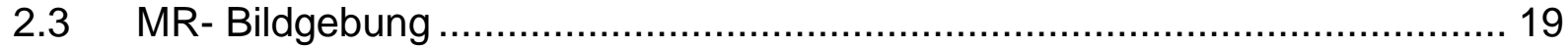

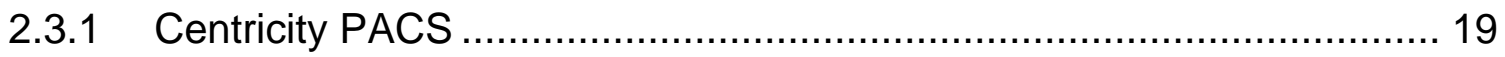

2.3.2 MRT des Kniegelenkes - wie die Bildaufnahmen entstehen ............... 19

2.4 Durchführung der Validitätsstudie ................................................... 20

2.5 Erhebung des posterioren Offsets der Femurkondyle............................. 21

2.6 Konstruktion der funktionellen Flexionsachse im Knie und Berechnung der Abweichung dieser zur sTEA, aTEA und PCA in Grad. ............................. 22

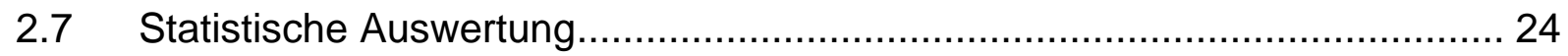

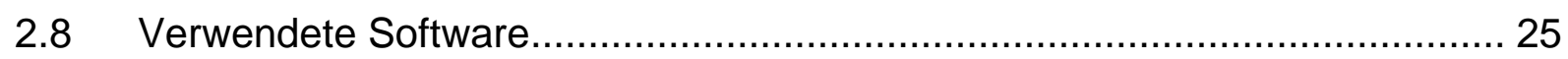

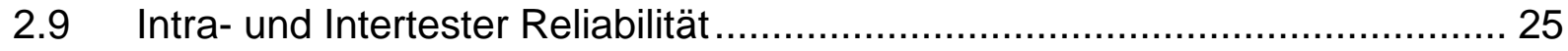

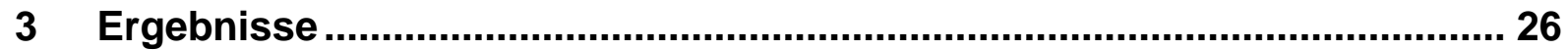

3.1 Auswertung primäres Patientenkollektiv .......................................... 26

3.1.1 Ergebnisse Validitätsstudie ................................................ 26

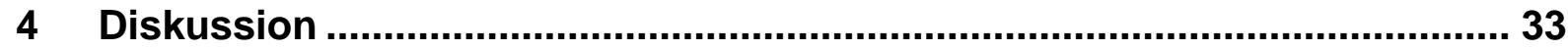




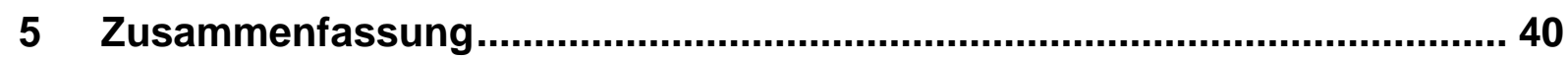

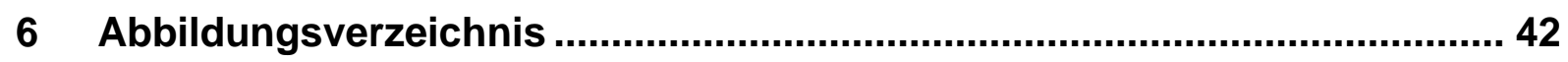

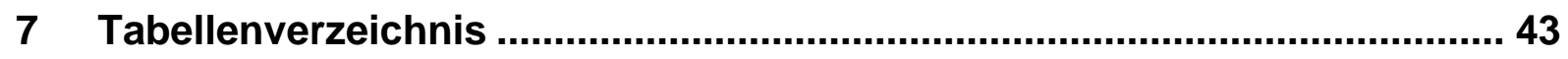

8 Literaturverzeichnis ..................................... Fehler! Textmarke nicht definiert. 


\section{$1 \quad$ Einleitung}

\section{$1.1 \quad$ Anatomie des Kniegelenks}

\subsubsection{Knöcherne Strukturen des Kniegelenks}

Das Kniegelenk ist das größte Gelenk des menschlichen Körpers. Seine knöcherne Grundlage bildet der Oberschenkelknochen (Os femoris) sowie das Schienbein (Tibia) und die Kniescheibe (Patella). Bei genauer Betrachtung ist das Kniegelenk ein zusammengesetztes Gelenk aus zwei Einzelgelenken, dem Kniescheibengelenk (Articulatio femoropatellaris), hierbei bilden Patella und Os femoris eine funktionelle Einheit, und dem für diese Arbeit entscheidendere Gelenk, dem Kniekehlgelenk (Articulatio femorotibialis), welches aus dem distalen Teil des Os femoris und dem Schienbeinkopf (Caput tibiae) besteht (Platzer 2009). Anatomisch gesehen zählt auch das Gelenk zwischen Wadenbein (Fibula) und Tibia (Articulatio tibiofibularis) zum Kniegelenk. Das distale Femurende zeigt nach medial und lateral zwei stark konvexe Kondyle (Gelenkknorren) auf, den Kondylus lateralis femoris und den Kondylus medialis femoris. Beide sind durch die Fossa interkondylaris voneinander getrennt. Zwischen den beiden Kondyle zeigt sich dorsalwärts die interkondyläre Notch, die sogenannte Kreuzbeinhöhle. Die Kondyle besitzen jeweils noch einen kleinen vorspringenden knöchernen Aufsatz, den Epikondylus medialis und lateralis. Unterhalb des lateralen Epikondylus befindet sich eine kleine Mulde, der sogenannte Sulcus popliteus. Die Kondyle sind beidseits mit Gelenkknorpel überzogen, was einen schmerzfreien Bewegungsablauf ermöglicht.

Der zweite Knochen, die Tibia, die an der Articulatio femorotibialis beteiligt ist, ist wie das Femur ein Röhrenknochen. Die Tibia besitzt ebenso an ihrem Caput tibiae cranial zwei überknorpelte Kondyle die in der Mitte durch eine knöcherne Erhöhung der Eminentia interkondylaris in einen lateralen und einen medialen Anteile getrennt werden. Die Area interkondylaris anterior und posterior begrenzen diese und stellen den Befestigungspunkt für die Kreuzbänder und Menisken dar. 


\subsubsection{Ligamentäre Strukturen des Kniegelenks}

Die beiden Kreuzbänder dienen dem Zusammenhalt beider Gelenkkörper. Sie verhindern das Abgleiten der Femurkondyle von den flachen Gelenkpfannen des Tibiaplateaus in die jeweilige Richtung. Zusammen mit den Kollateralbändern bilden sie mit anderen statischen Elementen wie Knochen, Kapsel und Menisken ein Gerüst. Gemeinsam wird das Gelenk mit dynamischen muskulären Strukturen stabilisiert und der Bewegungsumfang festgelegt (Fu 1994). Zudem verfügen die Bänder über eine Mehrzahl von Mechanorezeptoren mit überwiegend propriozeptiver Funktion (Adachi 2002). In Abbildung 1 sind die Kreuz- und Seitenbänder schematisch dargestellt. Das Lig. cruciatum anterius (LCA) hat seinen Ursprung an der lateralen Wange der Fossa interkondylaris femoris und zieht von dort in sich verdreht zur Area interkondylaris anterior. Ein Teil der Fasern inseriert am vorderen Abhang der Eminentia interkondylaris und strahlt in das Vorderhorn des Meniscus medialis ein. Zudem ist das LCA in zwei funktionell gebündelte Anteile untergliedert (Amis und Dawkins 1991). Auf diese Weise erhält das Kniegelenk beim Bewegungsablauf zusätzliche Stabilität und ist bis zu einem gewissen Grad der Belastung vor Extrembewegungen geschützt (Furman 1976). Das Lig. cruciatum posterius (LCP) verläuft von der medialen Wange der Fossa interkondylaris zur Area interkondylaris posterior und windet sich dabei etwas um das LCA nach dorsal. In 80\% der Fälle strahlt ein laterales Bündel, das Lig. meniscofemorale posterius („Wrisberg“), nach distal zum hinteren Umfang des Meniscus lateralis aus. Seltener zieht ein Bündel zum vorderen Umfang des Meniscus lateralis, das als Lig. meniscofemorale anterius (,Humphrey“) bezeichnet wird (Putz 2007). Ebenso wie das LCA setzt sich das LCP aus zwei großen Bündeln zusammen (Hughston 1980). Auch hier tritt die Bedeutung der Anatomie unter Berücksichtigung der jeweiligen Funktion in den Vordergrund.

Weitere wichtige ligamentäre Strukturen sind die Menisken, sie dienen der Vergrößerung der Kontaktfläche zwischen den stark gekrümmten Femurkondyle und der flachen Gelenkpfanne des Tibiaplateaus (Abbildung 2). Der C-förmige Meniscus medialis ist mit seinem Vorder- und Hinterhorn über kurze Bänder mit dem Knochen im Bereich der Areae interkondylares anterior und posterior verankert und somit kaum verschiebbar. Gleichzeitig ist er mit dem Lig. collaterale mediale (LCM) fest verwachsen und wird dadurch bei einem Trauma des LCM oft in Mitleidenschaft gezogen.

Der Meniscus lateralis gleicht eher einem Dreiviertelring. Sein Ursprung und Ansatz liegen nahe beieinander im Bereich des Tuberculum interkondylare lateralis. Eine 
zusätzliche Bandbefestigung wie beim Meniscus medialis ist nicht vorhanden. Somit ist der Meniscus latereralis leichter beweglich und kann Belastungen einfacher ausweichen. Beide Menisken sind insgesamt verschiebbar und fungieren als transportable Gelenkfläche. Sie fangen 30-35\% der Druckbelastung im Kniegelenk auf (Schiebler und Korf 2007). Die vorderen Umfänge sind durch einen runden Faserzug, das Lig. transversum genus, miteinander verbunden. Dieses liegt eingebettet im Hoffa-Fettkörper (Putz 2007).

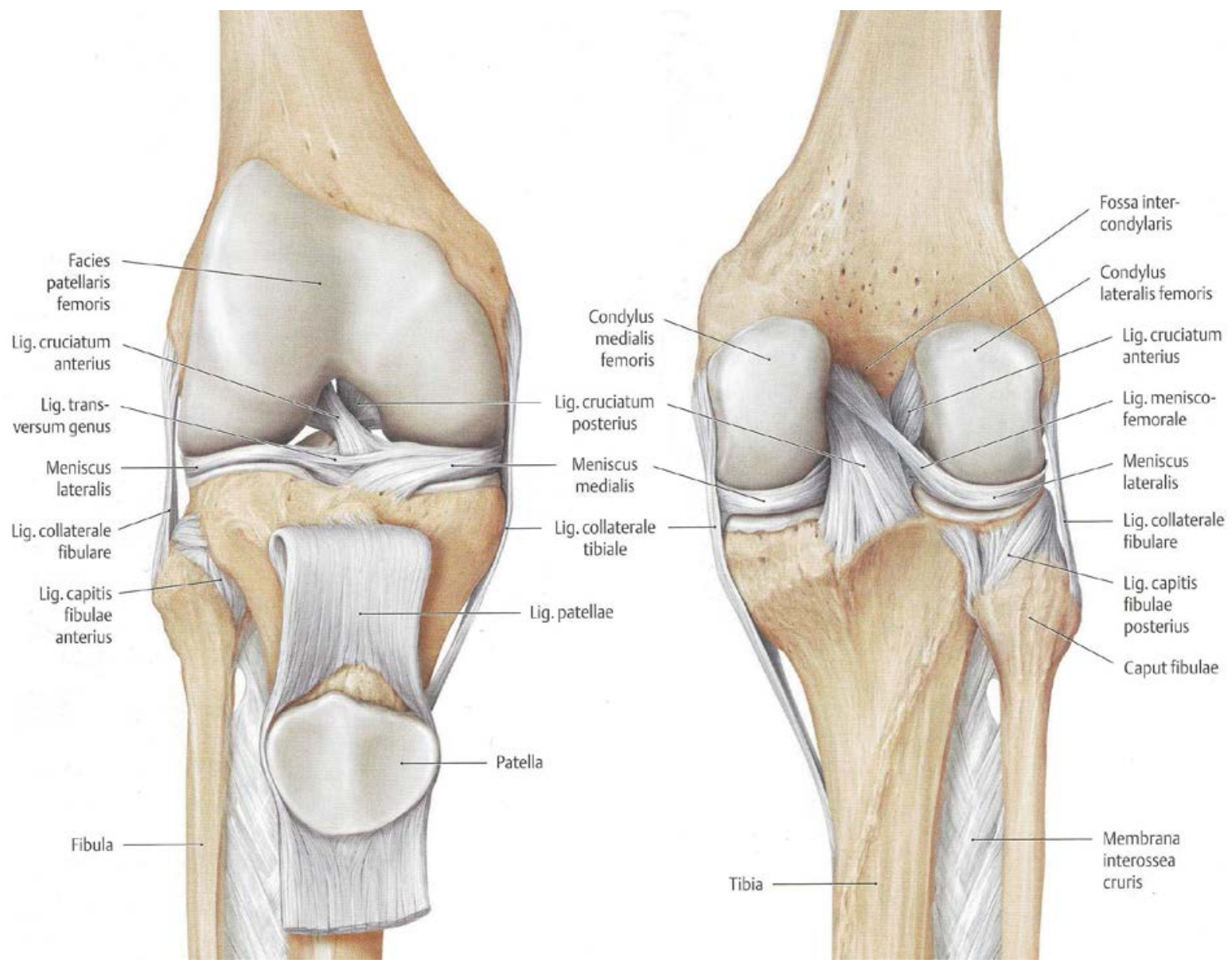

Abbildung 1: Schematische Darstellung eines rechten Kniegelenks mit den zugehörigen Bändern. Ansicht in Extension von ventral (links) und dorsal (rechts) (Schünke 2005). 


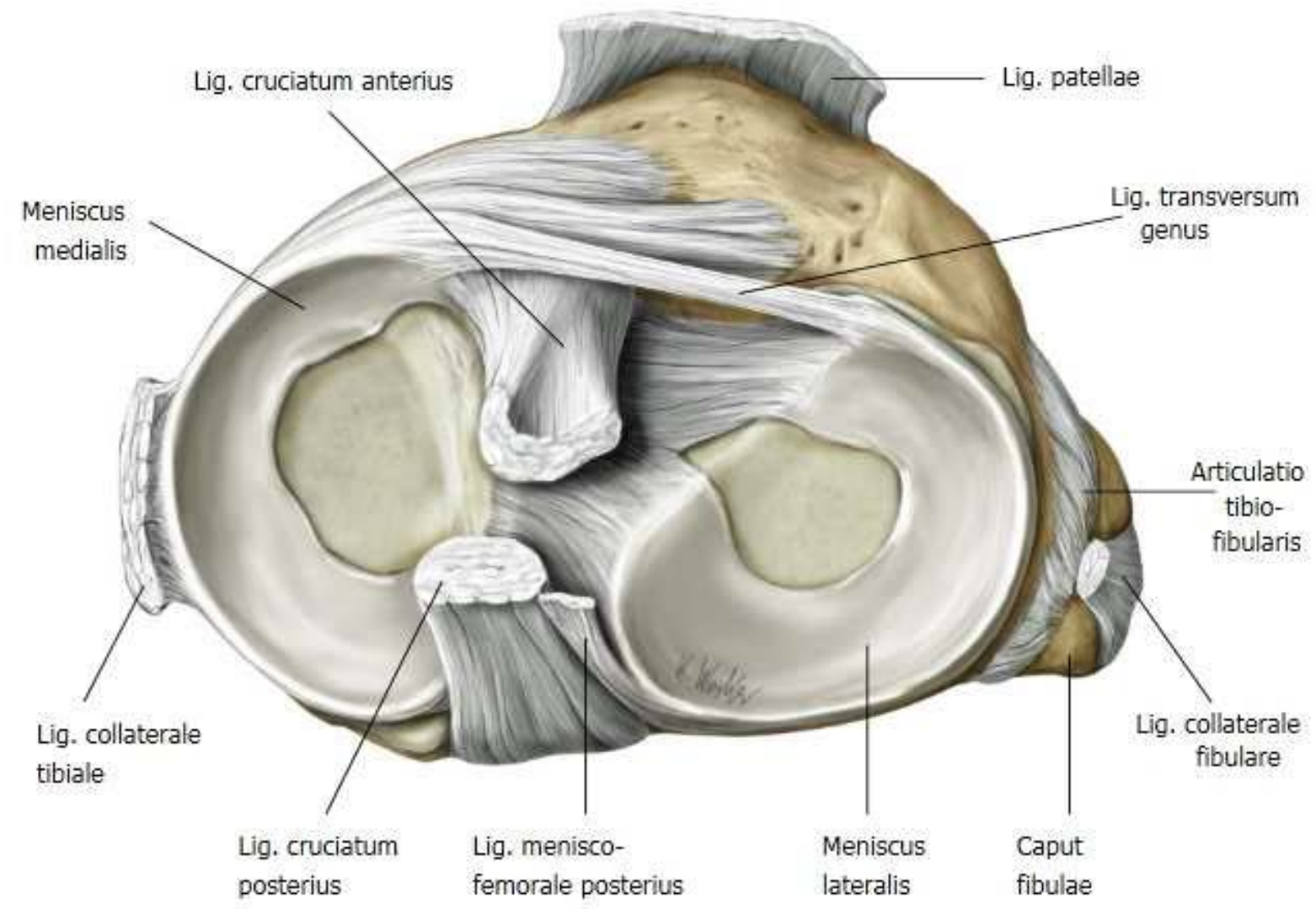

Abbildung 2: Die Menisken im rechten Kniegelenk von kranial (Schünke 2005).

\subsubsection{Genetische Entwicklung des Kniegelenks}

Tardieu et al. haben in ihrer Arbeit zeigen können, wie sich vor allem die femoralen Kondyle im Laufe eines menschlichen Lebens verändern (Tardieu 2006). Tardieu et al. beschreiben die genetische Entwicklung der Femurkondyle eines Neugeborenen, das eine eher quadratisch geformte Kondyle im Seitenprofil besitzt, hin zu einer deutlich nach dorsal gestreckten abgerundeten Kondyle. Wie weit aber die Kondyle nach dorsal wächst, kann bisher noch nicht vorhergesagt werden. Den Messergebnissen von Cinotti et al. ist es zu entnehmen, dass eine Person in der Seitendifferenz sowie sowohl einseitig medial als auch lateral unterschiedliche Kondylenlängen (siehe Abbildung 5) besitzen kann (Cinotti 2012). Den meisten Einfluss auf die Ausformung und das elliptische Profil der femoralen Kondyle hat im Laufe der Evolution des Menschen der aufrechte Gang. Für den aufrechten Gang ist die Anwesenheit eines bikondylären Winkels im Kniegelenk nötig (Shefelbine 2002). Dies ist der Winkel zwischen der Diaphyse des Oberschenkelknochens und einer Linie, die senkrecht in der infrakondylären Ebene verläuft. Shefelbine et al. zeigen, 
dass schon bei Ausgrabungen im Skelett des 3,5 Mio. Jahre alten Australopithecus afarensis im Knie ein solcher Winkel nachweisbar war, was für einen Gang auf zwei Beinen sprechen würde. Tardieu et al. untersuchten den bikondylären Winkel radiographisch an knapp 100 Probanden, sowohl weiblichen als auch männlichen Geschlechts, im Alter von einem Monat bis 17 Jahren. Tardieu zeigte in seiner Follow-up-Studie, dass der bikondyläre Winkel sich in der körperlichen Entwicklungsphase bis zur Adoleszenz von $0^{\circ}$ bis hin zu maximal $8^{\circ}-14^{\circ}$ verändert was direkt auch mit dem aufrechten Gang in Verbindung gebracht wurde, denn der größte Anstieg dieses Winkels erfolgte in der Zeit des Gehenlernens (Tardieu und Damsin 1997). Eine weitere Veröffentlichung von Igbigbi et al., die an 260 Malawiern untersuchte, wie sich die Femurkondyle bis in die Adoleszenz entwickeln, beschrieb auch, wie die laterale Trochlea des Femurs sich durch die Belastung des aufrechten Ganges prominenter entwickelt als die mediale Trochlea, was an den biomechanischen Kräften im Stehen liegt (Igbigbi 2005). Die unterschiedliche Ausformung der Femurkondyle hat wiederum Einfluss auf die individuelle Biomechanik im Kniegelenk der einzelnen Person und kann auch hier zu einem instabilen Gang führen (Dargel 2011).

\subsection{Biomechanik des Kniegelenks}

\subsubsection{Beinachse und Kniebewegung}

Von großer Bedeutung für die untere Extremität ist die geometrische Lagebeziehung der beteiligten Strukturen. Bei normalen Achsenverhältnissen liegen die Zentren des Hüft-, Knie- und Sprunggelenks auf einer Geraden. Diese stellt die mechanische Längsachse, die sogenannte Traglinie des Beins dar. Dagegen beschreibt die anatomische Achse den genauen Verlauf des Femur (Femurschaftachse) und der Tibia (Tibiaschaftachse). In der Orthopädie wird die Femurachse als "Mikulicz-Linie" bezeichnet (Schiebler und Korf 2007). Sie verbindet das Drehzentrum des Caput femoris, die Eminentia interkondylaris tibiae und das Zentrum der Malleolengabel am distalen Ende des Unterschenkels. Beim Tibiaschaft stimmen anatomische und mechanische Längsachse überein. Dagegen schließt die mechanische Achse des Femur mit der Femurschaftachse einen Winkel von etwa $6^{\circ}$ ein. Auf diese Weise liegen die Längsachsen von Ober- und Unterschenkel nicht auf einer Geraden. Stattdessen sind sie auf Höhe des Kniegelenks in der Frontalebene mit einem Winkel von $174^{\circ}$ 
nach außen abgewinkelt (Schünke 2005). Zu den Achsenabweichungen in der Frontalebene zählen das Genu varum (O-Bein) und Genu valgum (X-Bein) (Wülker 2005). Im ersten Fall liegt die Mitte des Kniegelenks lateral, im zweiten Fall medial von der Belastungslinie. Entsprechend der Fehlstellung ändern sich die Druck- und Kraftverhältnisse im Gelenk.

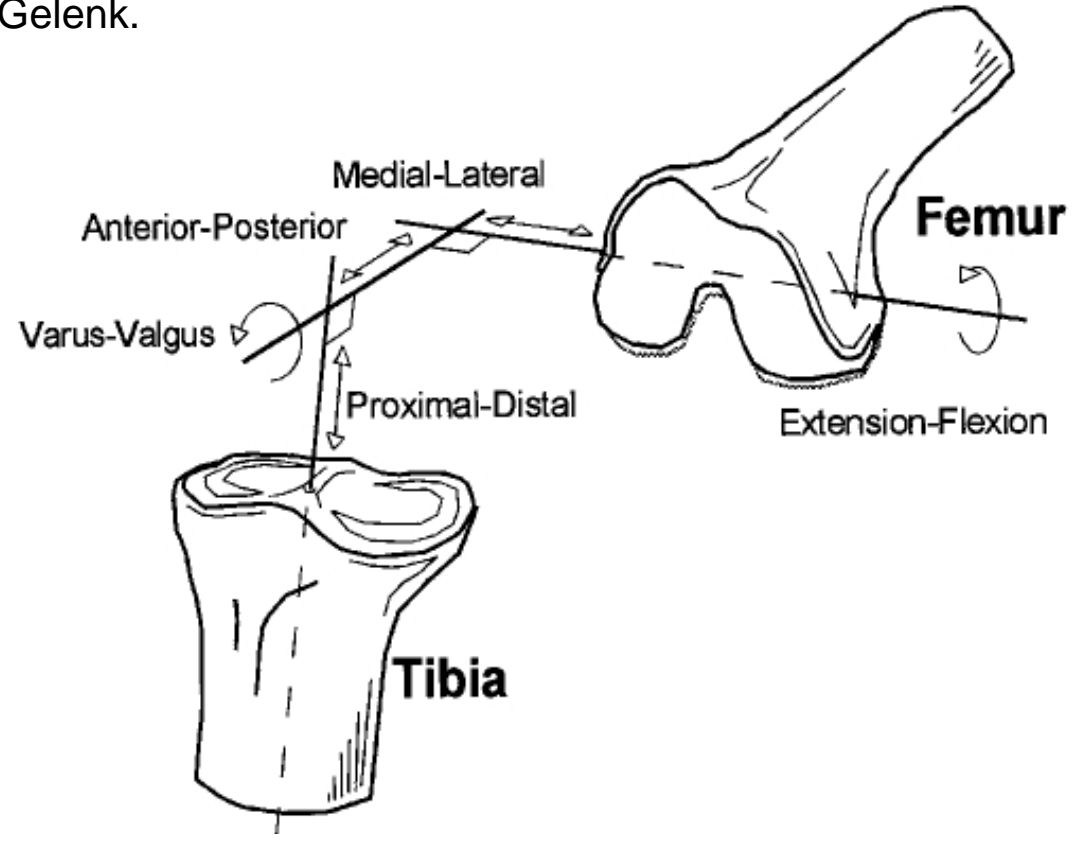

Abbildung 3: Die sechs Bewegungsgrade im Kniegelenk (Wülker 2005).

Den Freiheitsgrad eines Gelenkes kann man gedanklich einer Koordinate im dreidimensionalen Raum gleichsetzen. Zur Beschreibung einer Bewegung im Kniegelenk nutzt man die tibiale (longitudinale) Achse und jeweils dazu zwei senkrecht verlaufende Achsen. Die obige Abbildung 3 zeigt die sechs möglichen Freiheitsgrade im Kniegelenk, daraus definieren sich die folgenden möglichen Translations- und Rotationsbewegungen (Woo 1999):

(1) Sagittalachse: Adduktion / Abduktion (Varus / Valgus)

(2) Sagittalachse: Anterior- / Posteriortranslation

(3) Longitudinalachse: Innenrotation / Außenrotation

(4) Longitudinalachse: Distraktion / Kompression

(5) Transversalachse: Flexion / Extension

(6) Transversalachse: Medial- / Lateraltranslation

Der vierte aufgeführte Freiheitsgrad ist mathematisch berechnet und auch in der Literatur aufgeführt (Essinger 1989; Hanson 2006). Jedoch nehmen im Klinikalltag die Bewegungsrichtungen Flexion / Extension und Innenrotation / Außenrotation den größten Stellenwert ein. Zur Erfassung des individuellen Bewegungsumfanges eines 
Patienten wird die standardisierte Neutral-Null-Methode verwendet. Folgende Werte beschreiben den Normalbefund (Wülker 2005):

- Flexion / Extension: $150^{\circ}-0^{\circ}-0^{\circ}$

- Innenrotation / Außenrotation: $10^{\circ}-0^{\circ}-30^{\circ}$

\subsubsection{Bewegungsführung}

Damit es zu einer Bewegung in einem Gelenk kommt, sind einwirkende Kräfte in definierter Richtung, Kraft und Angriffspunkt die Voraussetzung. In Abhängigkeit davon wird durch den resultierenden Kraftfluss eine Bewegung ermöglicht. Die Übertragung an der gelenkigen Verbindung erfolgt durch eine Kombination von Druck senkrecht auf die Gelenkoberfläche und Zug in die umliegenden Weichteile. Die Zugspannung verläuft dabei entlang der Faseranordnung (Goodfellow und O'Connor 1978). Eine wesentliche Eigenschaft der Bandstrukturen ist die relative Unelastizität beziehungsweise geringe Dehnbarkeit. Die fixe Länge verhindert eine übermäßige Beweglichkeit des Gelenks und dadurch auch eine Dislokation (Woo 1999). Bei der Übertragung von Femur auf Tibia treten kaum signifikante Scherkräfte auf. Der Hauptgrund dafür liegt in der Beschaffenheit des hyalinem Knorpels, der auf Grund seiner Beschaffenheit über einen sehr niedrigen Reibungskoeffizienten von 0,005 - 0,025 verfügt (Santavirta 1998).

Aufgrund der lateral stark inkongruenten Gelenkflächen ist eine Knochenführung zur Vermeidung von Extrembewegungen nicht möglich. Eine geordnete Bewegungsführung wird hingegen durch das Zusammenspiel des gesamten Kapsel-Band-Apparats wirksam. Für eine möglichst homogene Kraftverteilung sorgen die beiden Menisken, die dank ihrer Faseranordnung und Form Krafteinwirkungen und Stöße abdämpfen können (Renström und Johnson 1990). Sie verleihen dem Kniegelenk die nötige Stabilität und optimieren durch ihre Lage im Kniegelenk die Kongruenz beider Gelenkflächen. Weiterhin sind sie für die Kraftübertragung zwischen den beteiligten knöchernen Strukturen Femur und Tibia zuständig (Goodfellow und O'Connor 1978; Walker und Erkman 1975). Das synergistische oder antagonistische Zusammenwirken verschiedener Muskeln und deren Einteilung in funktionelle Muskelgruppen sorgen für die zielgerechte Bewegung im Gelenk. Abhängig von ihren Ursprungsfeldern und ihrem Verlauf sind sie für die jeweilige Bewegung verantwortlich. Die Oberschenkelmuskeln werden daher topographisch und funktionell in eine vordere und hintere Muskelgruppe unterteilt. Die subkutan liegende derbe Fascia lata geht auf 
Höhe des Kniegelenks in die Fascia cruris über. Die Unterteilung der Muskelgruppen in drei Kompartimente erfolgt durch die Septa intermuscularia femoris laterale, mediale und posterius. Der lateral verlaufende Tractus iliotibialis verstärkt zusätzlich die Faszie und sichert das Kniegelenk. Durch Herabsetzen der auftretenden Zugspannung erhöht sich die Belastbarkeit des Femurs (Prinzip der Zuggurtung).

\begin{tabular}{|l|l|}
\hline Name & Funktion im Kniegelenk \\
\hline M. sartorius & Innenrotation \\
\hline M. rectus femoris* & Extension \\
\hline M. vastus intermedius* & Extension \\
\hline M. vastus lateralis* & Extension \\
\hline M. vastus medialis* & Extension \\
\hline M. articularis genus & Kapselspanner \\
\hline
\end{tabular}

Tabelle 1 Extensoren des Oberschenkels.

Die mit *) markierten Muskeln werden unter dem Begriff M. quadriceps femoris zusammengefasst und bilden die gemeinsame Quadricepssehne. Darin eingefügt liegt als Sesambein die Patella. Das Lig. patellae bezeichnet die Fortsetzung der Sehne bis zur Insertion an der Tuberositas tibiae.

Die Flexoren entspringen gemeinsam mit Ausnahme des Caput breve des M. biceps femoris am Tuber ischiadicum. Der M. biceps femoris inseriert am Caput fibulae und begrenzt mit seiner Ansatzsehne den lateralen Rand der Fossa poplitea. Der M. semitendinosus und $\mathrm{M}$. semimembranosus ziehen zum Pes anserinus beziehungsweise zum Kondylus medialis tibiae. Durch ihren Verlauf werden sie auch als ischiocrurale Muskulatur bezeichnet.

\begin{tabular}{|l|l|}
\hline Name & Funktion im Kniegelenk \\
\hline M. biceps femoris, caput longum & Flexion, Außenrotation \\
\hline M. biceps femoris, caput breve & Flexion, Außenrotation \\
\hline M. semitendinosus & Flexion, Innenrotation \\
\hline M. semimembranosus & Flexion, Innenrotation \\
\hline
\end{tabular}

Tabelle 2: Die Flexoren des Oberschenkels. 


\subsubsection{Kniegelenks-Kinematik}

Die Form der beiden Femurkondylen ist nicht identisch. Zusätzlich ist zu beachten, dass die laterale und mediale Femurkondyle sich in Abhängigkeit vom Flexionswinkel an unterschiedlichen Positionen auf dem medial konvexen und lateral konkaven Tibiaplateau befindet. Anstatt über das Tibiaplateau zu rollen, gleitet der mediale Kondylus in Form einer Rotationsbewegung um die transversale Achse nach dorsal. Dies bedeutet, dass bei zunehmender Flexion die posterioren Anteile des Femurkondylus auf der posterioren Gelenkfläche liegen. Entgegen früherer Meinungen ist nur eine verminderte posteriore Translationsbewegung des tibiofemoralen Kontaktpunktes um 2 mm zu verzeichnen (Iwaki 2000; Pinskerova 2000). Im lateralen Kompartiment liegt der vordere Anteil der Femurkondyle in voller Extension im vorderen Bereich des Tibiaplateaus. Aufgrund der ventral und dorsal deszendierenden Ausformung des lateralen Tibiaplateaus ist der laterale Meniskus dabei von Bedeutung. Seine Hörner füllen die Zwischenräume beider artikulierender Knochen aus und stabilisieren zusätzlich das Gelenk in dieser Position. Bei zunehmender Flexion im Knie erfährt der laterale Kondylus eine kombinierte Roll- und Gleitbewegung entlang der tibialen Artikulationsfläche nach dorsal. Entsprechend ist der tibiofemorale Kontaktpunkt am Ende der Flexionsbewegung um $2 \mathrm{~mm}$ nach posterior verschoben (Pinskerova 2000; Iwaki 2000). Bei der Kniebeugung legt der laterale Kondylus insgesamt eine längere Strecke zurück als der mediale Kondylus. Diese Differenz bewirkt zusätzlich eine passive Innenrotation der Tibia, die mit zunehmendem Flexionswinkel steigt. Die longitudinale Drehachse befindet sich dabei im Bereich des tibiofemoralen Kontaktpunktes im medialen Kompartiment (Schroeder-Boersch 2001). In gleicher Weise rotiert das Femur bei vermehrter Extension passiv nach innen (Pinskerova 2000). Dieser Vorgang wird in der Literatur als Screw Home- Mechanismus beschrieben (Andriacchi 1986). Bedingt durch die anatomischen Unterschiede beider Kompartimente im Frontalschnitt kommt es mit zunehmendem Flexionswinkel zusätzlich zu einer Varus Abweichung. Diese Adduktionsbewegung führt zu einem geringfügigen Aufklappen der Tibia nach lateral. Diese Besonderheit wird als lift-off- Mechanismus bezeichnet (Freeman und Pinskerova 2005). 


\subsection{Besonderheiten des distalen Femurs mit Bezug zur Kniegelenksendoprothetik}

\subsubsection{Femurkondyle}

Betrachtet man die Femurkondyle in sagittaler Schnittführung, erkennt man unterschiedliche Krümmungsradien. Hierbei erfolgt auch eine funktionelle Einteilung in das Flexionszentrum dorsal, in Abbildung 4 schematisch im 2. \& 3. Quadranten und dem Extensionszentrum ventral.

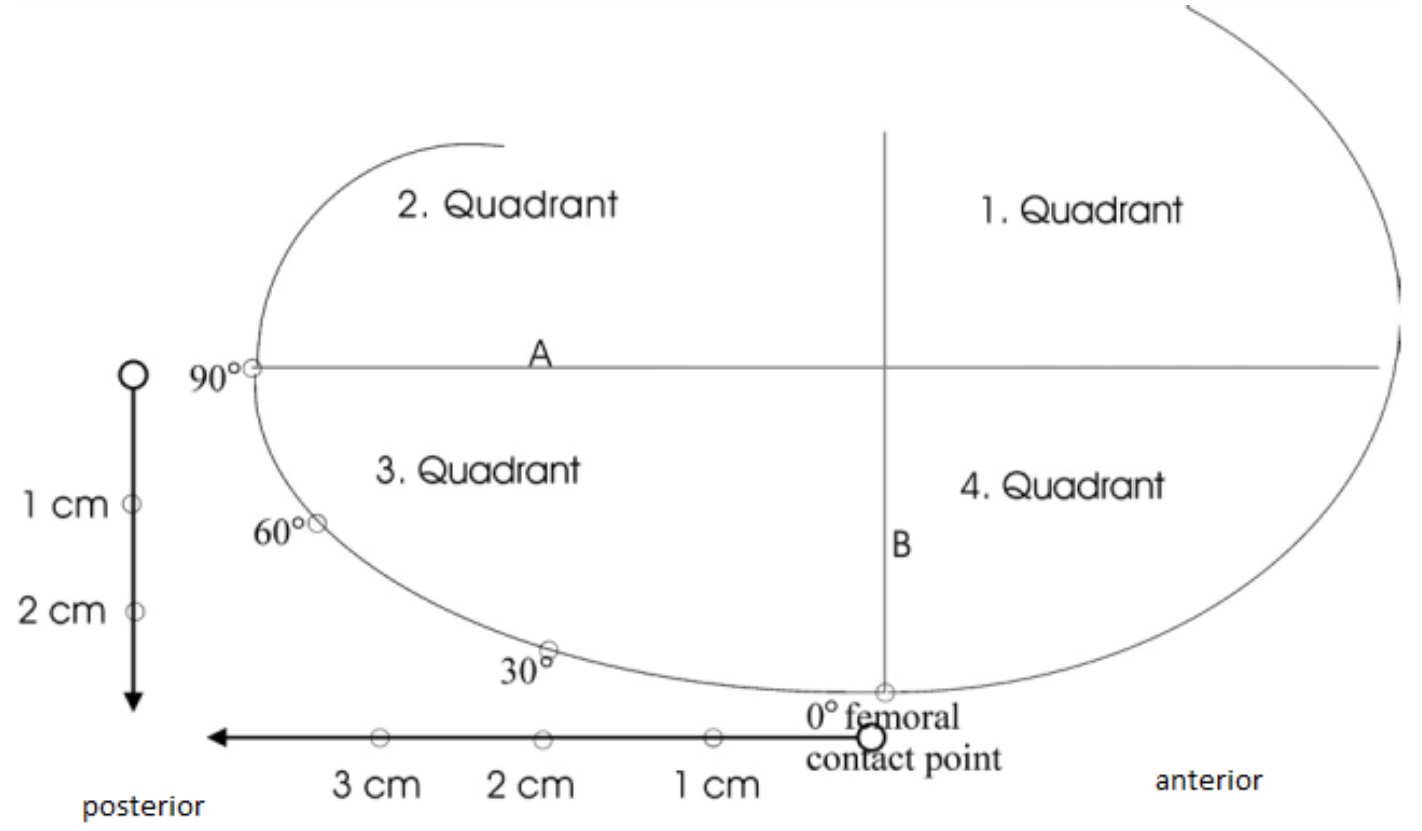

Abbildung 4: Schematischer Sagittalschnitt durch eine Femurkondyle (Biscević 2005).

Abbildung 4 zeigt die unterschiedliche Krümmung der Femurkondyle. Weiterhin ist in der Abbildung 4 der femorale Kontaktpunkt bei $0^{\circ}$ Extension eingezeichnet. Dies beschreibt die Situation bei gestrecktem Knie. Schematisch dargestellt sind entlang der Außenlinie des 3. Quadranten die weiteren Kontaktpunkte bei entsprechender Flexion im Kniegelenk bei $30^{\circ}, 60^{\circ}$ und $90^{\circ}$.

Biscević und Essinger zeigten jeweils in ihren Arbeitsgruppen die besondere Form der Femurkondyle, die dafür nötig sind, eine effiziente Bewegung im Kniegelenk zu ermöglichen und damit ein einheitlichen Gangbild zu erzielen (Biscević et al. 2005; Essinger et al. 1989). Wie bereits beschrieben, sind beide Femurkondylen nicht identisch und die Bewegung unterliegt einem komplexen Vorgang. Hierbei können sich mediale und laterale Femurkondyle nicht nur in Größe und Krümmung unterscheiden sondern - wichtig für diese Arbeit - es können auch deutliche Unterschiede im 
„Femoralen Offset“ zum Tragen kommen. Anatomisch beschreibt das KondylenOffset den weitesten Abstand zwischen der Projektion des posterioren femoralen Kortex (PFC) und der dorsalen Kante der Femurkondyle im rechten Winkel zueinander (Abbildung 5).

Kommt es zu einem fehlerhaft implantierten Gelenkersatz, sind vermehrt aseptische Lockerungen der Prothesen zu erwarten, die eine Revisionsoperation notwendig machen (Mulhall 2006). Weiterhin führt eine fehlerhafte femorale Rotation zu einer Instabilität im Femorpatellargelenk und zu einer schmerzhaften Instabilität der Kollateralbänderund des Kniegelenkes (Balcarek 2013; Hofmann 2003). Konnten Studien zeigen, dass vor allem die ausreichende Beugung im Kniegelenk ein wichtiger Outcome-Parameter nach erfolgter Operation ist, kann ein Patient nach fehlerhaft implantiertem totalem Kniegelenksersatz sein Knie nur noch eingeschränkt bewegen (Ritter und Campbell 1987). Ist die Beugung eingeschränkt, führt dies häufig zu einem nicht zufriedenstellenden postoperativen Ergebnis. Dies ist immer dann auch besonders der Fall, wenn der Patient die Beweglichkeit benötigt, um seinen kulturellen Gewohnheiten nachzukommen (Ritter 2008). Die unvorteilhafteste Situation wäre es, wenn eine Knieflexion unter $110^{\circ}$ das Resultat der Operation wäre. Jenes Winkelmaß wurde als dasjenige beschrieben, welches für die mindeste Mobilität und Aufgaben des alltäglichen Lebens benötigt wird (Kettelkamp et al. 1970; Laubenthal et al. 1972).

Um oben genannte Probleme zu vermeiden, ist eine sorgfältige präoperative Diagnostik mittels Bildgebung essentiell notwendig, diese sollte vor Implantation eines Kniegelenkersatzes erfolgen.

Von Cinotti et al. stammt auch die Untersuchungstechnik zur Messung des femoralen Offsets im MRT (Cinotti 2012). Cinotti zeigte in seinen Untersuchungen an 80 Probanden, dass der sagittale tibiale Anstieg (Englisch: sagittal tibial slope) stärker in Knien variiert als die femoralen Offsets. Einen Zusammenhang zum sagittal tibial slope konnte allerdings nur mit dem medialen Kompartiment hergestellt werden. Allerdings ist Cinotti auf mögliche Ursachen der Fehlmessung im MRT noch auf die durch die große Varianz bedingten Variationen der Flexionsachsen eingegangen und ihre damit verbundenen Auswirkungen auf die Implantation von Kniegelenksprothesen. Kommt es postoperativ hier zu einem unbalanciertem Flexionsspalt im Kniegelenk, sind Schmerzen, Patientenunzufriedenheit, Bewegungseinschränkungen bis hin zu Prothesenlockerungen die Folge (Ritter 2008; Hofmann 2003; Mulhall 2006). 


\subsubsection{Flexionsachsen}

\subsubsection{Die funktionelle Flexions- / Extensionsachse}

Das herkömmliche Verständnis der Kniegelenkskinematik ging davon aus, dass das Knie keine feste Flexions- / Extensionsachse besaß. Dies beruhte auf Beobachtungen in der Sagittalebene, in denen sich anfangs zeigte, dass sich das unmittelbare Rotationszentrum im Knie während des Flexionsvorganges innerhalb des hinteren Teils der Femurkondyle bewegte. (Frankel 1971; Soudan 1979). Im Gegensatz dazu zeigten neuere Studien, basierend auf dreidimensionalen Beobachtungen an Tierkadavern, dass die Flexion- und Extensionsbewegung im Knie besser beschrieben werden können als eine Bewegung, die sich um eine feste Flexions/Extensionsachse dreht und sich in dem hinteren Teil der Femurkondyle befindet (Abb. 6). Hierbei liegt die funktionelle Flexionsachse so, dass sie im distalen Femur jeweils durch die Mitte der posterior medialen und der posterior lateralen Femurkondyle verläuft (Hollister 1993; Stiehl 1995).

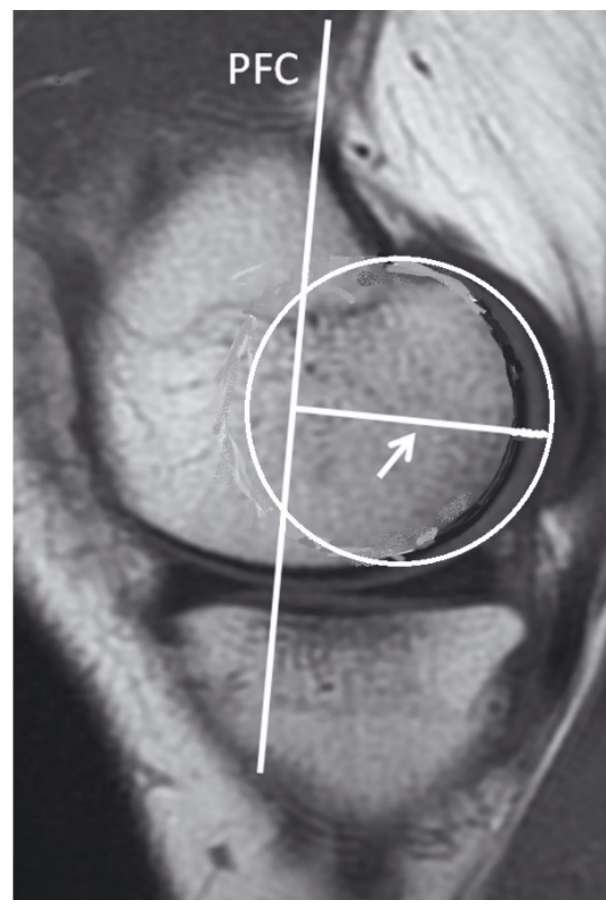

\section{Abbildung 5: Berechnung des femoraler Offsets.}

Sagittaler MRT-Scan mittig durch eine Femurkondyle. PFC als Projektion des femoralen Kortex in der Mitte des Femurkondyle. Hier beschreibt der Kreis den Krümmungsradius des Flexionszentrums. Der weiße Pfeil zeigt das femorale Kondylen-Offset. 

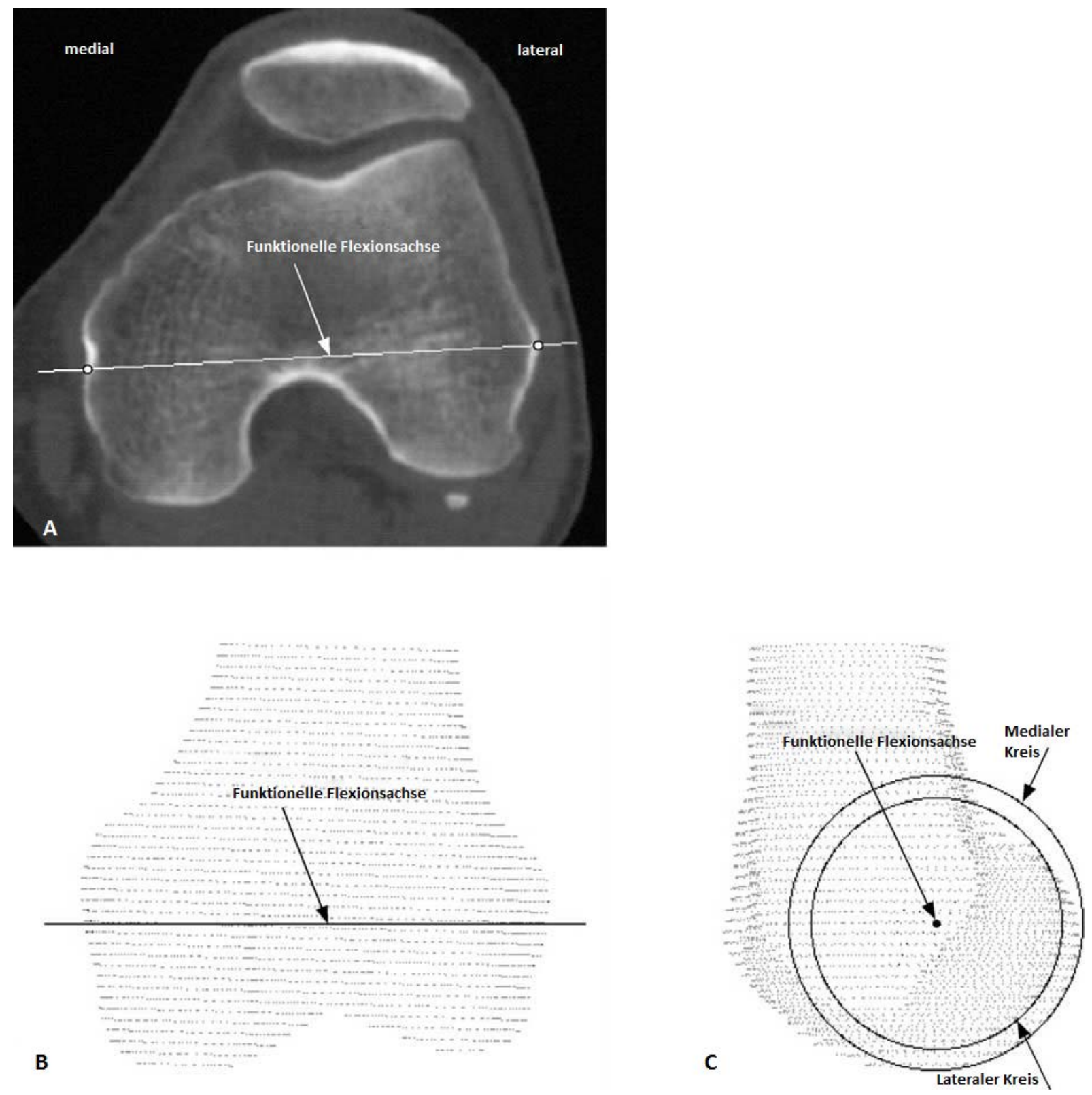

Abbildung 6: Die funktionelle Flexionsachse

(A) Funktionelle Flexionsachse projiziert auf einen Transversalschnitt im CT (B) In frontaler Aufsicht (C) Betrachtung der Femurkondyle in seitlicher Ansicht; Kreise umkreisen den Verlauf der medialen und lateralen Femurkondyle.

\subsubsection{Transepikondyläre Achsen und posteriore kondylen Achse}

Die Geometrie der proximalen Tibia und das distale Femur sind eng mit der Biomechanik des Tibiofemoral- und Patellofemoralgelenks verknüpft. Im Themengebiet der Kniegelenksarthroplastik spricht man bei der Positionierung des Implantates in der axialen Ebene auch von einer rotationsgerechten Angleichung (Engl.: rotational alignment) eines solchen. Ein Knieimplantat fungiert als Oberflächenersatz für das umliegende Weichteilgewebe. Infolgedessen beeinflusst die genaue rotatorische Po- 
sitionierung des Implantates das postoperative Ergebnis. Jegliche rotatorische Fehlplatzierung hat Auswirkungen auf die Belastung der angrenzenden Gelenkflächen und die Zugspannung der beteiligten Bandstrukturen (Victor 2009). Dies führt wiederum zu abweichendem kinematischem Verhalten, welches Steifigkeit, Instabilität des Knies bis hin zur vorzeitigen Lockerung der Prothese zur Folge hat. Eine symptomatische Flexionsinstabilität bedingt durch einen übergroßen Flexionsspalt auf der medialen Knieseite ist die Folge einer starken Außenrotation des eingebrachten Femurimplantats (Olcott 1999). Miller et al. wiesen nach, dass vergrößerte Scherkräfte auf die Kniescheibe wirken, wenn der femorale Anteil des Implantates nicht richtig eingebracht wurde (Miller 2001). Skolnick et al. waren die ersten, die den Zusammenhang eines intraoperativ korrekt ausgerichteten Implantates mit dem daraus klinischen Nutzen für die Patienten herstellen konnten (Skolnick 1976). Mit Hilfe eines ,Röntgenindex' gelang es Lotke und Ecker die Ausrichtung des tibialen und fermoralen Kompartiments zu beschreiben und einen signifikanten Zusammenhang zwischen korrekt positioniertem Implantat und positivem postoperativem Ergebnis herzustellen (Lotke 1977). Er schlug auch vor, weiter Anstrengungen in die Entwicklung neuer standardisierter Operationsverfahren zu investieren. Seit dieser Zeit war man ständig bemüht, Methoden und konventionelle Achsen zu beschreiben, die ein rotationsgerechtes Ausrichten des Implantates ermöglichen.

Transepikondyläre Achsen wurden in der Vergangenheit eingehend auf ihre zuverlässige Verwendbarkeit im distalen Femur untersucht. Jene im Besonderen als eine nützliche mögliche Referenzachse, die benötigt wird, um in der totalen Kniegelenksarthroplastik (TKA) mögliche Fehlrotationen zu ermitteln und zu verhindern (Stiehl 1995; Mantas 1992; Berger 1992; Matsuda 1998). So gilt die Erzeugung eines parallelen, balancierten Beugespaltes als eine der Voraussetzungen in der Knieglenksendoprothetik, dies bezieht auch die Bestimmung der korrekten femoralen Rotation mit ihren Referenzachsen mit ein. So zeigten Miller et al. (Miller 2001), dass in der totalen Kniegelenksendoprothetik es die Artikulation im tibiofemoralen und patellofemoralen Gelenk im höchsten Maße fördert, wenn man den femoralen Anteil des Gelenkersatzes parallel zur transepikondylären Achse einbringen würde. Es waren Berger et al. (1992), die bereits vorher in ihrer Arbeit zwei transepikondyläre Achsen ermittelten: Die anatomische transepikondyläre Achse (aTEA) und die chirurgische transepikondyläre Achse (surgical TEA; sTEA). 


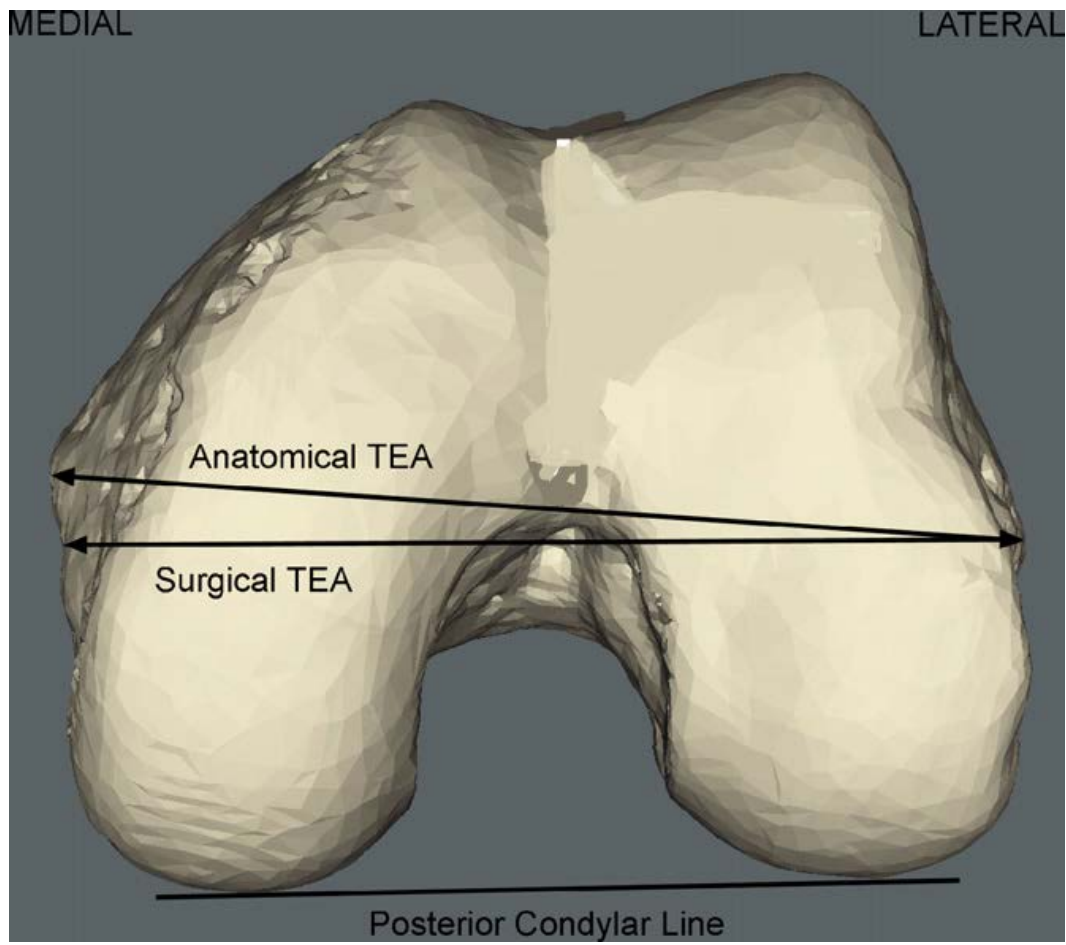

Abbildung 7: Femur in axialer Ebene, mit den Referenzachsen in der Kniegelenksendoprothetik Aufsicht auf den distalen Femur in axialer Ebene, mit den für die Femurrotation wichtigen Referenzachsen in der Kniegelenksendoprothetik (Victor 2009).

Die aTEA verbindet den knöchernen Überhang der medialen Kondyle mit dem knöchernen Vorsprung der lateralen Kondyle. Die STEA verbindet ebenfalls den Vorspung der lateralen Kondyle mit dem Sulcus unterhalb des medialen Vorsprungs, an dem die tiefer gelegenen Fasern des medialen Kollateralbandes entspringen. Des Weiteren beschreibt die posteriore kondylen Achse (PCA), in Abbildung 7 als ,Posterior Kondylar Line' dargestellt, eine Tangente zwischen den beiden posterioren Kondylen.

Alle Achsen weisen jedoch Schwächen bezüglich ihrer Verlässlichkeit untereinander auf. Mantas verglich die PCA mit der aTEA und zeigte, dass je nach angewendeter Referenzachse Unterschiede in der femoralen Rotation auftraten von bis zu $\pm 4,9^{\circ}$ (Mantas 1992). Beim Vergleich der sTEA mit der PCA von Griffin zeigten sich Abweichungen von durchschnittlich $6^{\circ}$ (Griffin 2000). Yoshioka et al. versuchten die STEA und die aTEA miteinander zu vergleichen, jedoch gelang es nur in $30 \%$ der Fälle überhaupt, die konventionellen Achsen intraoperativ ausfindig zu machen, die benötigt wurden, um beispielsweise den Sulcus medialis zu identifizieren (Yoshioka 1987). Alle Studien zeigten Schwächen bei der Durchführung zur Ermittlung der Achsen, 
dies galt besonders intraoperativ. Hier zeigte sich die PCA als beste zu identifizierende Achse. Asano et al. veröffentlichten mit Bezug auf Miller et al., dass die sTEA die ideale Referenzachse darstellt, die verwendet werden soll, um eine TKA auszurichten (Asano 2005; Miller 2001).

Alle verwendeten Achsen wurden allerdings nur zweidimensional ohne Beachtung eines weiteren Einflussfaktors untersucht, des femoralen Kondylen Offsets. Zeigten doch, wie in Kapitel 1.3.1 erwähnt, die Untersuchungen von Cinotti et al., dass das femorale Kondylen Offset Einfluss auf den Flexionsspalt hat (Cinotti 2012). Bis zu diesem Zeitpunkt wurden die einzelnen Entitäten meist separat voneinander betrachtet, die Ergebnisse der Korrelation von tibial slope, femoralem Offset und Flexionsachsen sowie deren postoperativer Einfluss auf die Kniegelenksplastik wurden kontrovers diskutiert (Bellmans 2002, 2005; Massin 2006). Cinotti konnte dann 2012 zeigen, dass ein Zusammenhang zwischen tibaial slope und femoralem Offset besteht, hatte die Auswirkungen auf die Femurachsen jedoch nicht berücksichtigt (Cinotti 2012).

\subsection{Fragestellung}

Die Fachliteratur der Orthopädie und Biomechanik zeigt, dass bereits viele Anstrengungen unternommen wurden, die physiologische Kinematik des menschlichen Kniegelenkes detailliert zu untersuchen. Neben klinischen Studien nach Implantation einer Kniegelenkstotalendoprothese mit Evaluation der subjektiven und objektiven Ergebnisse existieren zahlreiche experimentelle Studien, die sich mit dem Bewegungsausmaß nach Kniegelenkersatz beschäftigen. Jedoch kommt es nach Einsetzen einer TKA immer wieder zu Einschränkungen im Bewegungsumfang des Kniegelenks, was wiederum zu unbefriedigenden klinischen Ergebnissen führt. In vielen Fällen ist eine eingeschränkte Beugefähigkeit auf eine Dysbalance des Seitenbandapparates in Flexion zurückzuführen. Im unzulänglich ausbalancierten Beugespalt der TKA kann es dabei zu einem einseitigen Kompressionsdruck auf das Polyethylen kommen. Ein erhöhter Abrieb mit evtl. Prothesenlockerung oder auch das subjektiv empfundene schlechte Ergebnisse aufgrund der verminderten Beugefähigkeit können zur einer Revisionsoperation führen (Laskin 1989). Neben der Dysbalance des Seitenbandapparates in Beugung kann auch eine Reduktion des distalen femoralen Offsets verantwortlich für eine schlechte Beugefähigkeit mit Reduktion der Patientenzufriedenheit sein (Bellemanns 2002; Massin 2006). Es stellt sich daher die Frage, 
ob die unbefriedigenden Ergebnisse einiger Patienten nach Implantation einer KnieTotalendoprothese möglicherweise auf eine individuelle Variabilität der funktionellen Flexionsachse oder des posterioren Kondylen-Offsets zurückgeführt werden können. Eine Variabilität, die durch die klassischen operativen Techniken möglicherweise nicht bemerkt bzw. nicht ausgeglichen werden kann. Es erscheint daher von besonderer Bedeutung, zunächst die Häufigkeit und die Streubreite, mit der Abweichungen von der anatomischen Norm angenommen werden müsse zu untersuchen. Dies bedarf wiederum einer genauen Analyse, die jedoch anhand der etablierten und standardisierten präoperativen Röntgenbilder des Kniegelenks nicht ausreichend möglich ist. Es bietet sich hier die Kernspintomographie als nicht strahlenbelastende mehrdimensionale Untersuchungsmethode an.

In der hier vorliegenden Arbeit werden dabei folgen Hypothesen aufgestellt:

1. Die Kernspintomographie stellt ein adäquates Verfahren zur verlässlichen präoperativen Bestimmung des distalen femoralen Offsets dar.

2. Die Größe bzw. die Differenz des medialen und lateralen femoralen Offsets ist individuell und zeigt eine große Streubreite.

3. Die in der Kniegelenksendoprothetik beschriebenen Rotationsachsen (aTEA, sTEA und PCA) korrelieren nur ungenügend mit der individuellen funktionellen Flexionsachse.

4. Das posteriore Kondylen-Offset hat einen statistisch signifikanten Einfluss auf die Genauigkeit der anatomischen Rotationsachsen (aTEA, sTEA und PCA) zur funktionellen Flexionsachse. 


\section{Probanden und Methoden}

\subsection{Ethische Fragen}

Die Durchführung dieser Studie wurde von der Ethikkomision der Universitätsmedizin Göttingen genehmigt (Antragsnummer: DOK_21_2014).

\subsection{Patientenkollektiv}

Die retrospektive Studie setzte sich zunächst aus 183 konsekutiven mitteleuropäischen Patienten zusammen. Dafür wurden im Zeitraum von März 2006 bis Juni 2010 insgesamt 87 rechte und 96 linke Knie von 88 Frauen und 95 Männern im Alter von 10 - 59 Jahren mit einem MRT untersucht. Die MRT-Untersuchung wurde in allen Fällen aufgrund eines klinischen Verdachts auf eine bestehende diskoligamentäre Pathologie durchgeführt. Zum Ausschluss aus der Studie führten vorangegangene Umstellungsosteotomien an Tibia oder Femur, Trochleadysplasie, Frakturen im distalen Femur, degenerative Gelenkerkrankungen oder schon ein durch eine Kniegelenksprothese versorgter Patient.

Durch eine Vorauswertung der Daten ergab sich der Verdacht, dass die beobachtete große Streuung des medialen und lateralen posterioren Kondylen-Offsets im Zusammenhang mit der verwendeten Messmethodik stehen könnte.

Um diesen Zusammenhang weiter zu untersuchen bzw. auszuschließen wurden eine Validitätsstudie mit 5 weiteren Probanden angeschlossen, die nicht ursprünglich zu dem oben genannten Kollektiv gehörten (Kapitel 2.4).

Die hieraus gewonnenen Erkenntnisse flossen in die weiteren Auswertungen mit ein, so dass von den ursprünglich 183 Probanden 127 Probanden von der weiteren Analyse ausgeschlossen wurden. Für die Beantwortung der Studienziele wurden schlussendlich 56 Kernspinuntersuchungen von 56 Probanden zur Messung des femoralen posterioren Kondylen-Offsets und der sich hieraus ergebenden Achsabweichungen durchgeführt.

Die 56 Kernspinuntersuchungen setzten sich aus 28 weiblichen und 28 männlichen Probanden im Alter zwischen 13 - und 59 Jahren zusammen. Insgesamt wurden 28 rechte und 28 linke Knie im Kernspintomographen vermessen. 


\section{$2.3 \quad$ MR-Bildgebung}

\subsubsection{Centricity PACS}

Centricity PACS ist eine skalierbare IT-Plattform für medizinisches Bilddatenmanagement. Alle Messungen wurden mit Hilfe dieser Software in der Version Centricity PACS 4.0 durchgeführt. Das Computerprogramm ist ein Produkt der GE Healthcare mit Sitz in Barrington, Illinois USA. Klare Vorteile dieses Programms sind das Einhalten der Industrienormen wie DICOM, HL-7, IHE und XDS, sowie die Verwendbarkeit von fortschrittlichen Bildbearbeitungswerkzeugen und dem sicheren Datenschutz der bearbeiteten Dateien (Centricity PACS Imaging 2013). Des Weiteren ermöglicht das Programm eine Präzise Messung der zu untersuchenden Winkel und Abstände, bis auf eine Stelle hinter dem Komma.

\subsubsection{MRT des Kniegelenkes - wie die Bildaufnahmen entstehen}

Die MRT-Untersuchung des Patientenknies erfolgt in Rückenlage. Das Knie des Patienten wird unterpolstert, fixiert und in die röhrenförmige Spule eingebracht.

Zu Beginn wird eine sogenannte Lokalizersequenz in allen drei Ebenen, ultrakurz (wenige Sekunden) und wenig auflösend mit einer T1-Wichtung, zur Raumorientierung und Planung der folgenden Untersuchung des Kniegelenkes durchgeführt. In der Regel beträgt die Standardschichtdicke hier $3 \mathrm{~mm}$. Die Einstellung der Schichten erfolgt an einer axialen Schicht, die durch das patellofemorale Gelenk angefertigt wird. An diesem Lokalizer werden dann die sagittalen Schichten rekonstruiert. 
Die transversale Schicht wird an der posterioren Kondylen Achse ausgerichtet und die sagittalen Schnitte erfolgen senkrecht darauf, mittig durch die Fossa interkondylaris. Das Anlegen dieser Achsen an die Lokalizersequenz in axialer Ausrichtung erfolgt durch die oder den medizinisch-technische/-n Radiologieassistentin/-en (MTRA). Bei der Ausrichtung des quadratischen Lokalizers (in der Abbildung 8 gelb dargestellt) kann der zu wählende Bereich nicht nur in dieser Schnittebene zweidimensional dargestellt, rechts oder linksdrehend um den Lokalizermittelpunkt (Abbildung 8: kleiner gelber Kreis), sondern auch einseitig verändert werden.

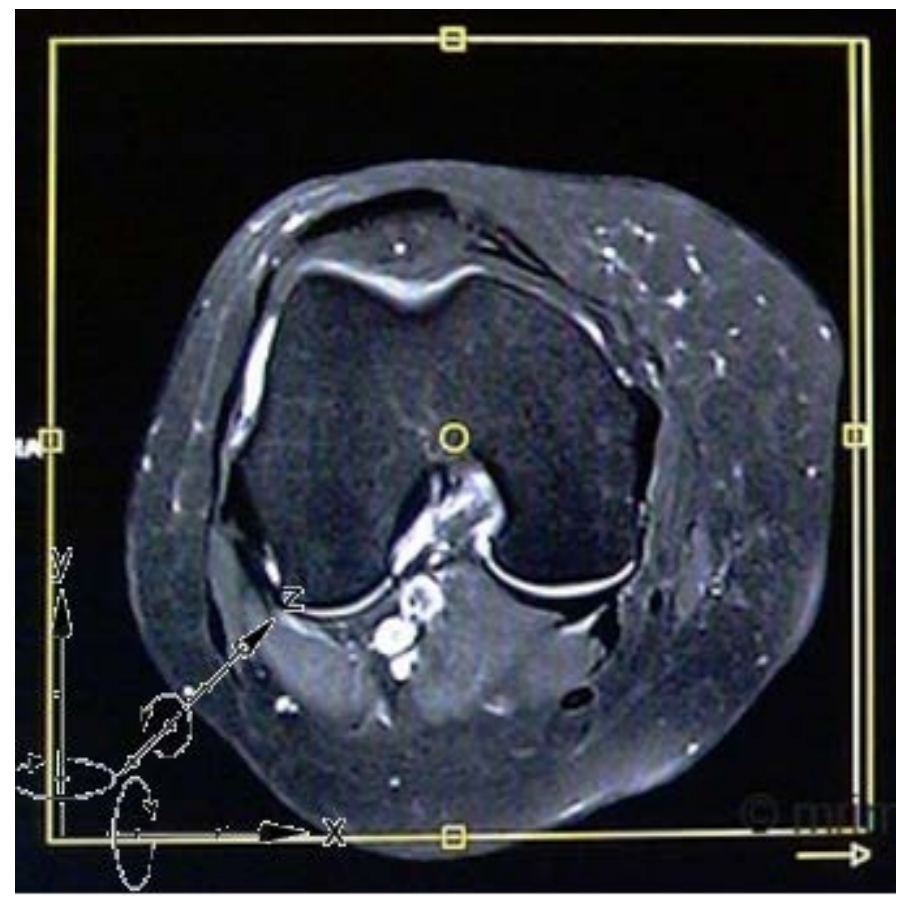

Abbildung 8: Ausrichten des Lokalizers an einer axialen Schicht im MRT.

Die Pfeile zeigen die Möglichkeit der Rotation des auszuwählenden Bereiches in alle drei Achsen.

\subsection{Durchführung der Validitätsstudie}

Es wurde angenommen, dass es zu erhöhten Messungenauigkeiten des femoralen Kondylen-Offsets kommen kann, wenn die sagittale Schicht nicht exakt $90^{\circ}$ zur aTEA ausgerichtet ist. Diese Position wurde als „Neutralposition“ definiert.

Es wurden erneut fünf gesunde Probanden untersucht. Alle fünf Probanden erhielten eine MRT-Bildgebung vom rechten und vom linken Knie. Pro rechtem und linken Knie wurden drei verschiedene Aufnahmen gefahren. Eine Aufnahme in „neutraler“ Position, d. h. $90^{\circ}$ zur aTEA, und jeweils eine Aufnahme in der der Lokalizer um $5^{\circ}$ 
nach innen- bzw. $5^{\circ}$ nach außen gedreht wurde. Hieraus entstanden für jeden Probanden sechs Einzeluntersuchungen seiner beiden Knie.

An der Bildgebung wurde erneut, wie bereits in Kapitel 2.5 beschrieben, das femorale posteriore Kondylen-Offset bestimmt.

Jedes Offset wurde viermal gemessen, insgesamt wurden 240 Messungen durchgeführt.

\subsection{Erhebung des posterioren Offsets der Femurkondyle}

Zur Ermittlung des posterioren-Offsets wurde die allgemein angewandte Technik von Cinotti et al wie folgt angewandt (Cinotti 2012): Um das posteriore Offset der Kondyle zu ermitteln wählt man im ersten Schritt im PACS denjenigen sagittalen MRT Schnitt der genau durch die Mitte des Kniegelenks und dem distalen Drittel der Femurdiaphyse geht. Es wird daraufhin die sagittale longitudinale Achse des Femurs konstruiert (Abbildung 9A). Nachdem diese als hintere femorale Kortexachse ermittelt wurde, wird diese jeweils nach medial und nach lateral in die Mitte der Femurkondyle verschoben. Zur systematischen Identifizierung desjenigen Sagittalschnittes, welcher die größte posteriore Ausdehnung der Femurkondyle zeigt (Abbildung 9B), wurde zur Hilfenahme ein äquivalenter Transversalschnitt verwendet. Hierüber wurde der zu verwendete sagittale Schnitt ermittelt, an dem später auch die Messung des femoralen Kondylen Offsets erfolgte. Im Sagittalschnitt wurde nun der am weitesten dorsal liegende Punkt auf dem posterioren Femurkondylenkortex identifiziert und von dort aus eine Gerade gezogen die im rechten Winkel auf die hintere femorale Kortexachse inklusive angrezendem Knorpel zieht (Abbildung 9C). Die Länge dieser Geraden wurde als das femorale Kondylen Offset in Zentimetern von dem Programm PACS auf eine Dezimale hinter dem Komma gerundet, für jeden Patienten medial und lateral gemessen. Für das posteriore Offset wurde auch das Verhältnis (Ratio) der medialen zur lateralen Kondyle berechnet. 

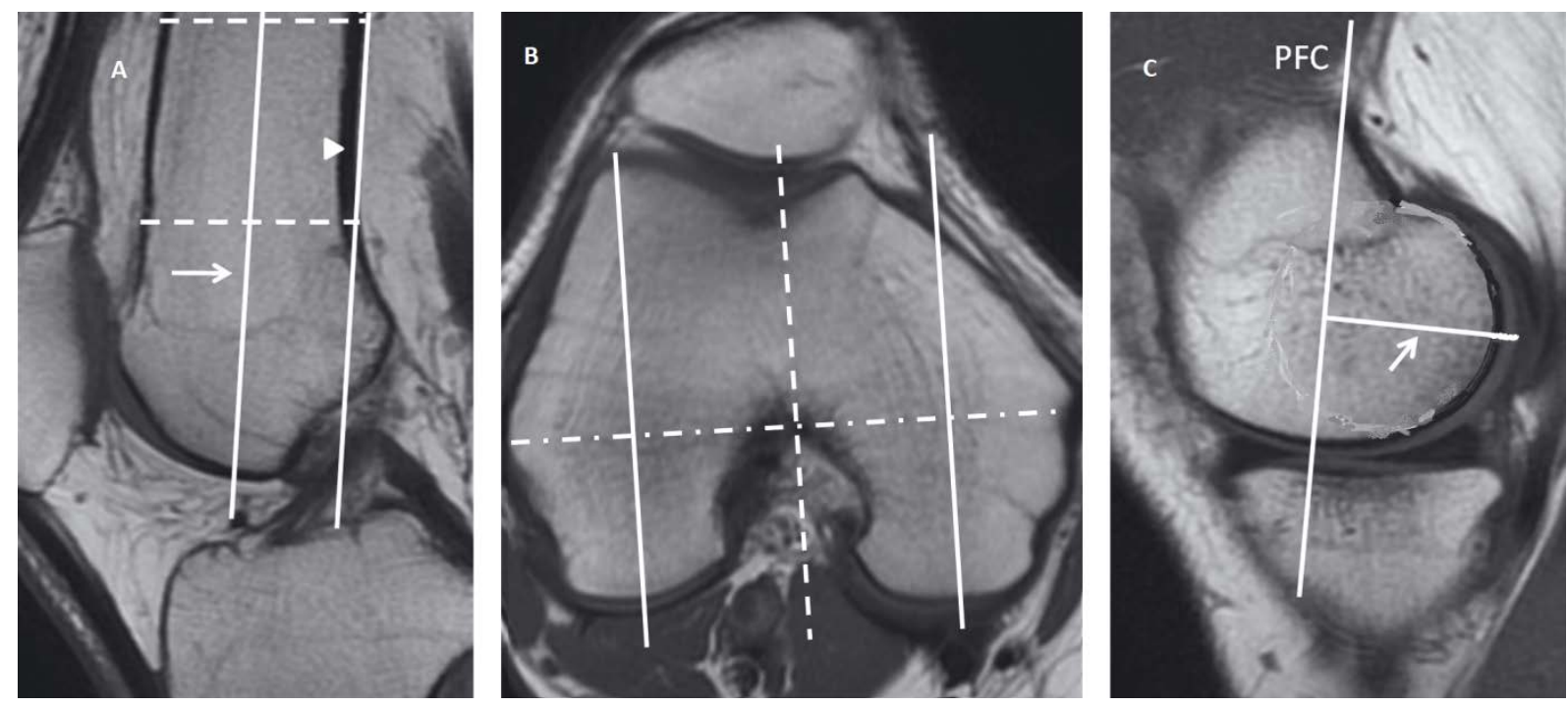

\section{Abbildung 9: Messung des posterioren Kondylen Offsets}

(A) Sagittalschnitt zur anschaulichen Darstellung der longitudinalen Femurachse (Pfeil) und der Verschiebung zum posterioren Femurkortex (Pfeilspitze). (B) Transversalschnitt der die größte Femurkondylenfläche zeigt. Die gestrichelte Linie zeigt den Sagittalschnitt von (A). Entlang der durch Striche und Punkte gekennzeichneten transepikondylären Achse wird die posteriore Femurkortexachse nach medial und lateral verschoben (durchgezogene Linien). (C) Sagittalschnitt mittig durch die Femurkondyle. Länge der Femurkondyle (Pfeil), rechtwinklig auf die Achse des posterioren Femurkortex (PFC) mit einschließlich femoralem Knorpelüberzug. (Bilder A und B aus Cinotti 2012)

\subsection{Konstruktion der funktionellen Flexionsachse im Knie und Berechnung der Abweichung dieser zur STEA, aTEA und PCA in Grad.}

Zur weiteren Analyse der Bilder wurden wieder die MRT-Sequenzen mit T1-Wichtung aus der transversalen und der sagittalen Schnittebene mit Hilfe eines Querverweises untereinander verknüpft. Ziel ist es nun, die funktionelle Flexionsachse durch die Mitte der lateralen und medialen Femurkondyle zu legen. Hierzu wird, wie bereits in 2.5 beschrieben, der Sagittalschnitt verwendet, der jeweils die größte posteriore Kondylenausdehnung zeigt. Im darauffolgenden Schritt wird in diesem Schnitt an die posteriore Kondyle ein Kreis angelegt, der möglichst genau außen auf dem Gelenkknorpel aufliegt und deren Form nachempfunden ist (Abbildung 10A). Jetzt wird mit zwei senkrecht aufeinander stehenden Geraden der Mittelpunkt dieses Kreises ermittelt. Hierbei dienen die Geraden auch als Autokorrektur des Kreises, sie sind hierbei stets gleich lang. Das jetzt verwendete Bildbearbeitungswerkzeug „3DCursor/Multiplanare Lokalisierung“ der PACS Software (Curser-Symbol in Abb. 
10A/10B) ermöglicht es durch Auswählen des Mittelpunktes des Kreises und der vorangegangenen Verknüpfung der Schnitte verschiedener Ebenen, den jetzt markierten Mittelpunkt auch in dem zugehörigen Transversalschnitt zu markieren. Wiederholt man den Vorgang für beide Seiten eines Knies, erhält man im Transversalschnitt zwei Punkte. Die Verbindungslinie dieser Punkte repräsentiert die individuelle funktionelle Flexionsachse des Kniegelenks. Die weiteren Schritte erfolgen jetzt am Transversalschnitt mit eingezeichneter funktioneller Flexionsachse. Die in der Einleitung beschriebenen konventionellen Achsen (aTEA, sTEA und PCA) werden bestimmt und eingezeichnet (Abb. 10B). Mit Hilfe der Winkelmessfunktion wurden jeweils die Abweichung der aTEA, sTEA und der PCA von der funktionellen Flexionsachse (fFA) bestimmt. Hierbei wurden für eine einheitliche Datenerhebung, Vermeidung von Folgefehlern und einer später besseren Nachvollziehbarkeit folgende Vorzeichen den Winkeln zugeordnet: Einer Abweichung in Grad der ermittelten Achse in Relation zur fFA in Innenrotation wurde ein negatives Vorzeichen zugeordnet. Dementsprechend wurde einer Abweichung der ermittelten Achse in Relation zur fFA in Außenrotation ein positives Vorzeichen zugeordnet.

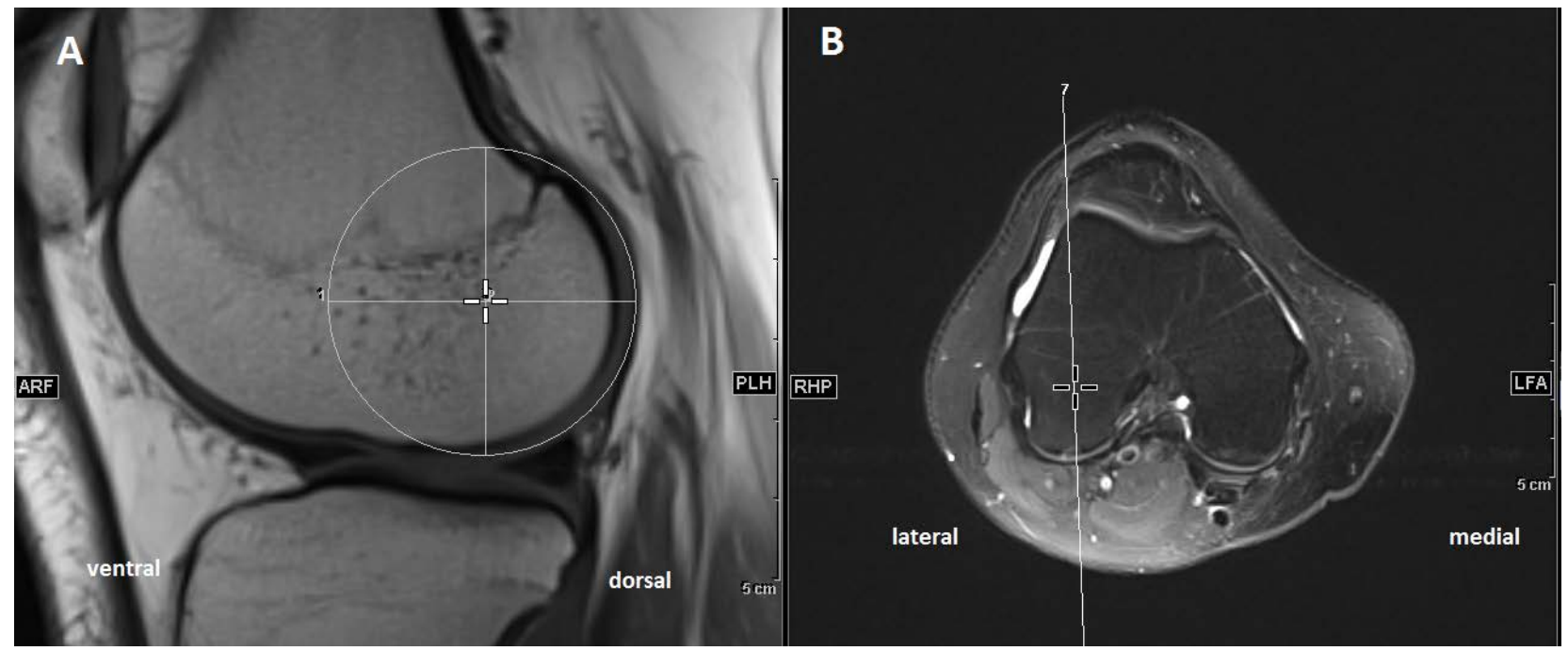

Abbildung 10: Berechnung der Rotationsachse in der posterioren Femurkondyle

(A) Die Größe des Kreises entspricht der Größe der posterioren Femurkondyle. Die senkrecht zueinander stehenden Geraden bilden den Mittelpunkt. Fadenkreuz ist das „3D-Cursor/Multiplanare Lokalisierung" -Werkzeug der Bildbearbeitung. (B) Die durchgezogene Linie zeigt die Schnittebene des Sagittalschnitts von (A). Das Fadenkreuz markiert identische Punkte in unterschiedlichen Ebenen. 

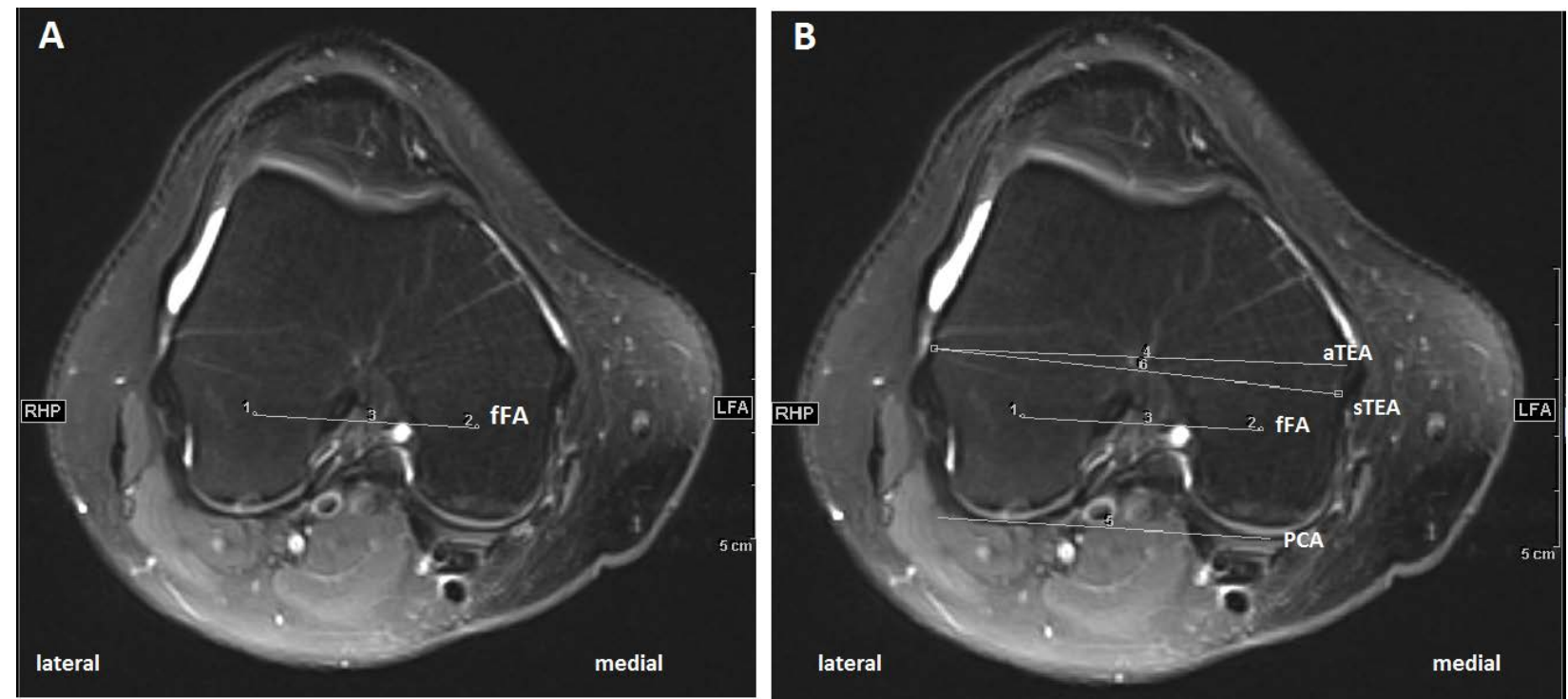

Abbildung 11: fFA im Verhältnis zu den konventionellen Achsen

MRT, Sagittalschnitt distaler Femur: (A) 1 und 2 markieren die Mittelpunkte der Femurkondyle. (B) Einzeichnung der einzelnen Achsen.

\subsection{Statistische Auswertung}

Das Institut für Medizinische Statistik der Universität Göttingen übernahm beratende Funktion bezüglich der statistischen Testverfahren und der Auswertung.

Ausgehend von den ermittelten Rohdaten werden die Abweichung der Winkel von STEA, aTEA und PCL in Abhängigkeit von der funktionellen Flexionsachse ermittelt.

Die Messwerte des Kondylen-Offsets werden als Mittelwert +/- Standardabweichung dargestellt. Die Abhängigkeit der Achsabweichungen von der fFA unter Berücksichtigung des Kondylen-Offsets wurde mit einer Varianzanalyse (ANOVA) durchgeführt. Die Korrelation zwischen den einzelnen Achsenabweichungen zu der Ratio aus medialen zu lateralen Kondylen Offsetlänge wurde mit Hilfe des PearsonsKorrelationskoeffizienten ermittelt. Ein $p<0,05$ wurde als statistisch signifikant angenommen. 


\subsection{Verwendete Software}

Verwendet wurde das Programm STATISTICA $®$, welches eine Statistik-Software des Herstellers StatSoft mit Hauptsitz in Tulsa im US-amerikanischen Bundesstaat Oklahoma ist. Statistica ist eine universelle Software für statistische und grafische Datenanalysen. Sie ist modular aufgebaut und bietet ein breites Spektrum an Methoden.

In der aktuellen Version STATISTICA $10 \AA$ finden sich grundlegende statistische Auswerteverfahren wie statistische Kennziffern, Korrelationen und Varianzanalyse.

\subsection{Intra- und Intertester Reliabilität}

Für die Bestimmung der Intertester-Reliabilität wurde oben verwendete Methodik zur Erfassung der Daten von zwei weiteren ärztlichen Kollegen, die nicht fachfremd waren, mit derselben Gruppe Patienten zeitgleich durchgeführt. 


\section{Ergebnisse}

\subsection{Auswertung primäres Patientenkollektiv}

In dem primär untersuchten Patientenkollektiv $(n=183)$ gab es eine große Variabilität in dem Ausmaß der Länge der einzelnen posterioren Femurkondylen-Offsets (PCO). In den MRT gestützten Messungen war das laterale Offset im Mittel aller Messungen $30,82( \pm 4,22) \mathrm{mm}$ ( $\min .14,3$ bis max. 41,7 mm), das mediale Offset im Mittel aller Messungen $31,76( \pm 4,32) \mathrm{mm}(\min .21,2$ bis max. 44,7 $\mathrm{mm}$ ) lang. Die mittlere Ratio des medialen posterioren Kondylen-Offsets zum lateralen Kondylen-Offset war 1,05 $( \pm 0,19)$. Allerdings lag die Bandbreite der einzeln errechneten Ratios zwischen 0,61 und 1,85, welches bei einer Ratio von 1,85 bedeutete, dass das mediale Offset 12,1 $\mathrm{mm}$ größer als das laterale Offset gemessen wurde.

Es zeigte sich bei der Datenauswertung, dass sowohl bei einer Ratio $>1$ aber auch bei einer Ratio von $<1$ eine vermehrte Innenrotation auftrat.

Aufgrund dieser sich darstellenden Diskrepanz bzw. großen Streubreite der PCO`s wurde mit 5 Probanden eine Validitätsstudie durchgeführt. Diese Probanden waren nicht Teil des ursprünglichen Kollektivs, hatten aber dieselben Ausschlusskriterien (siehe 2.4). Die Durchführung dieser Studie wurde von der Ethikkommission der Universitätsmedizin Göttingen genehmigt (Antragsnummer: 18/7/16).

\subsubsection{Ergebnisse Validitätsstudie}

Folgende Ergebnisse können nach Auswertung der MRT`s von den 5 Probanden dargestellt werden. In den MRT gestützten Messungen war das laterale Offset im Mittel aller Messungen 29,36 ( \pm 2,2) mm (min. 25,32 bis max. 34,92 mm), das mediale Offset im Mittel aller Messungen 31,88 ( \pm 3,29) $\mathrm{mm}(\min .27,6$ bis max. 40,17 mm) tief in Neutralposition (Tabelle 3).

Bei einer simulierten Innenrotation von $5^{\circ}$ der zu untersuchenden Extremität wurde die mediale PCO im Mittel mit 29,79 ( \pm 2,61) mm (min. 25,21 bis max. 35,06 mm) und das laterale PCO im Mittel mit 31,13 ( \pm 2,07) mm (min. 27,06 bis max. 34,66 $\mathrm{mm}$ ) lang gemessen (Tabelle 3).

Bei einer simulierten Außenrotation von $5^{\circ}$ wurde die mediale PCO im Mittel mit $33,71( \pm 3,15) \mathrm{mm}(\min .29,0$ bis max. 40,56 mm) und das laterale PCO im Mittel mit 27,6 ( $\pm 2,14) \mathrm{mm}$ ( $\min .24,03$ bis max. 32,21 mm) lang gemessen (Tabelle 3). 


\begin{tabular}{|c|c|c|c|c|}
\hline & Mittelwert & Minimum & Maximum & SD \\
\hline Mediales PCO in Neutralposition & $31,88 \mathrm{~mm}$ & $27,6 \mathrm{~mm}$ & $40,17 \mathrm{~mm}$ & $\pm 3,29 \mathrm{~mm}$ \\
\hline Mediales PCO bei $5^{\circ}$ Innenrotation & $29,79 \mathrm{~mm}$ & $25,21 \mathrm{~mm}$ & $35,06 \mathrm{~mm}$ & $\pm 2,61 \mathrm{~mm}$ \\
\hline Mediales PCO bei $5^{\circ}$ Außenrotation & $33,71 \mathrm{~mm}$ & $29,0 \mathrm{~mm}$ & $40,56 \mathrm{~mm}$ & $\pm 3,15 \mathrm{~mm}$ \\
\hline Laterales PCO in Neutralposition & $29,36 \mathrm{~mm}$ & $25,37 \mathrm{~mm}$ & $34,92 \mathrm{~mm}$ & $\pm 2,2 \mathrm{~mm}$ \\
\hline Laterales PCO in $5^{\circ}$ Innenrotation & $31,13 \mathrm{~mm}$ & $27,06 \mathrm{~mm}$ & $34,66 \mathrm{~mm}$ & $\pm 2,07 \mathrm{~mm}$ \\
\hline Laterales PCO in $5^{\circ}$ Außenrotation & $27,6 \mathrm{~mm}$ & $24,03 \mathrm{~mm}$ & $32,21 \mathrm{~mm}$ & $\pm 2,14 \mathrm{~mm}$ \\
\hline
\end{tabular}

Tabelle 3: Ergebnisse aller Messungen des medialen und lateralen posterioren kondylen Offsets (PCO) in Neutralposition, $5^{\circ}$ Innen- und $5^{\circ}$ Außenrotation.

Die nachfolgende Abbildung (Abbildung 12) zeigt grafisch die Abweichungen der gemessenen PCO`s in neutraler Position sowie nach $5^{\circ}$ Innen- und $5^{\circ}$ Außenrotation. Gemessen am Mittelwert wird in Neutralposition das mediale PCO 2,52 mm größer als das laterale PCO gemessen. Dieser Unterschied ist nicht signifikant ( $p$-Wert > $0,05)$.

Gemessen am Mittelwert wird bei $5^{\circ}$ Außenrotation die mediale Kondyle 1,83 mm größer, und die laterale Kondyle 1,76 mm kleiner im Vergleich zur Neutralposition gemessen. Dies bedeutet eine absolute Differenz von 3,59 mm. Dieser Unterschied ist signifikant ( $p$-Wert $<0,05)$.

Gemessen am Mittelwert wird bei $5^{\circ}$ Innenrotation die mediale Kondyle 2,09 mm kleiner und die laterale Kondyle 1,77 mm größer im Vergleich zur Neutralposition gemessen. Dies bedeutet eine absolute Differenz in der $5^{\circ}$ Innenrotation von 3,86 $\mathrm{mm}$. Dieser Unterschied ist ebenfalls signifikant ( $p$-Wert $<0,05)$. 


\section{A Mediale Kondyle}

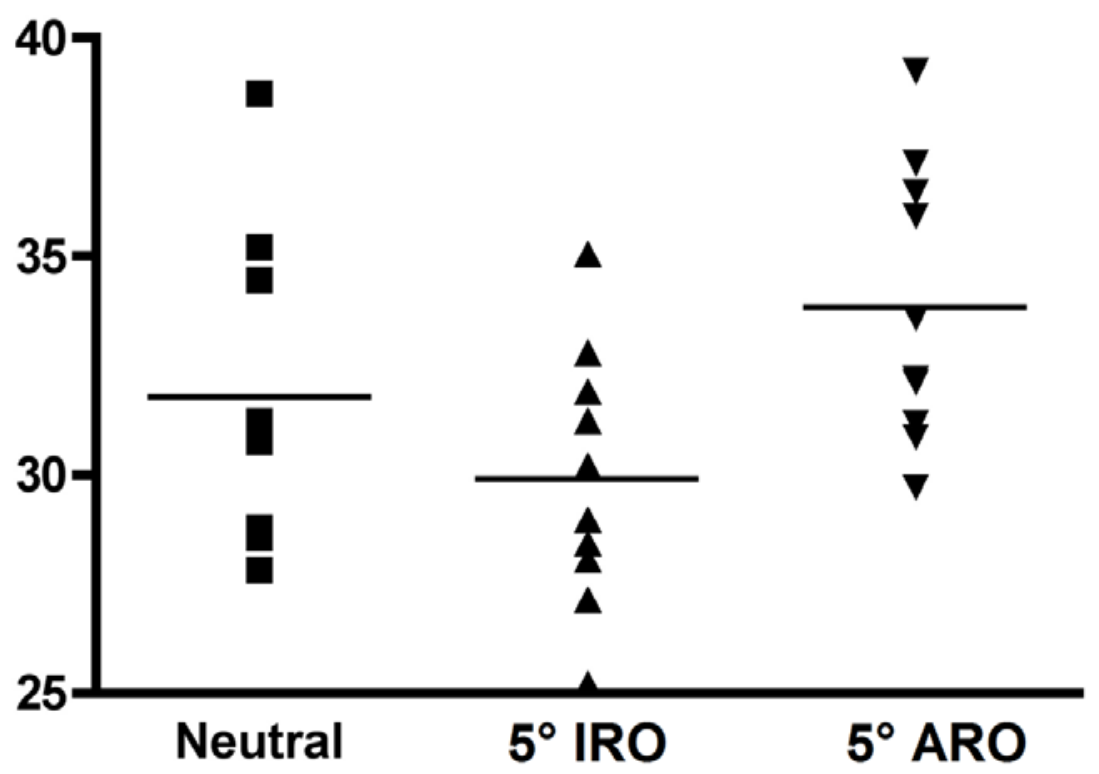

\section{B Laterale Kondyle}

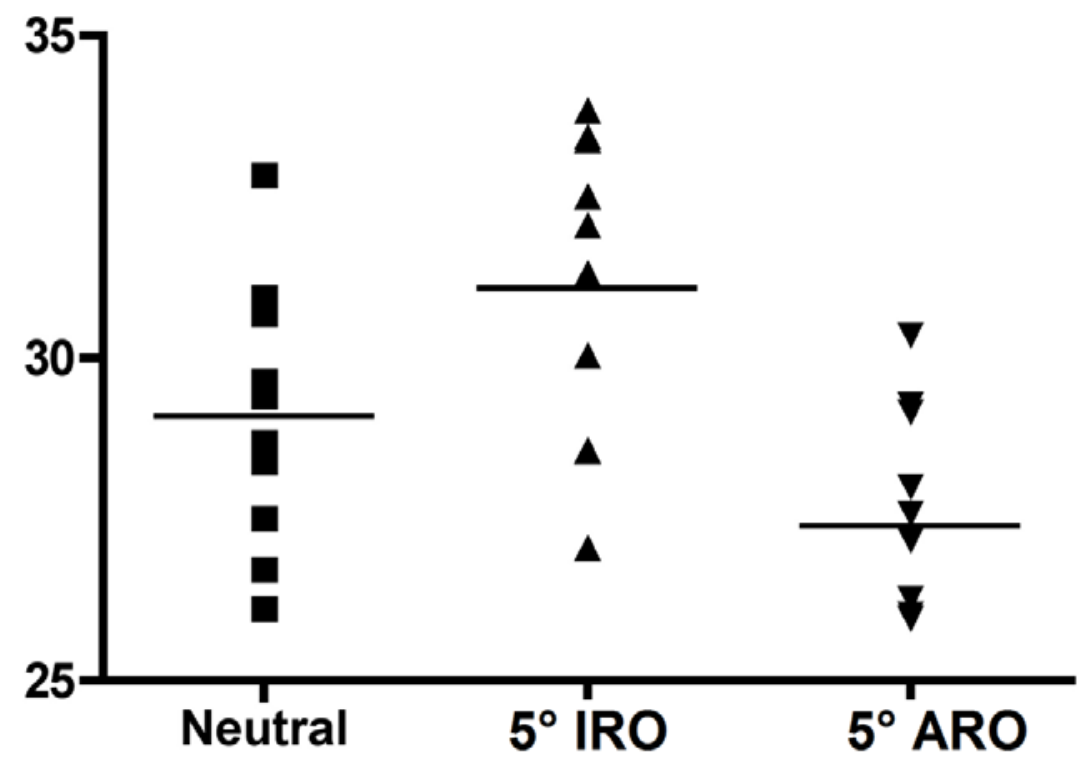

Abbildung 12: Abweichung der PCO-Länge bei unterschiedlichen Rotationsstellungen des Knies. Ergebnisse in $\mathrm{mm}$ (y-Achse) der gemessenen PCO an der medialen (A) und lateralen Femurkondyle (B). $x$-Achse: Jeweils in neutraler $0^{\circ}$-Position, $5^{\circ}$ Innenrotation ( $5^{\circ}$ IRO) und $5^{\circ}$ Aussenrotation ( $\left.5^{\circ} \mathrm{ARO}\right)$. Die waagrechte Linie entspricht dem Mittelwert. 
In Kapital 2.4 wurde definiert, dass eine Neutralposition angenommen wird, wenn die sagittale Schicht des zu untersuchenden Knies im MRT's exakt 90 zur aTEA ausgerichtet ist.

In der Validitätsstudie konnte ein signifikanter Einfluss der Rotation auf das Messergebnis dargestellt werden. Daher wurden die initialen 183 Patienten unter folgendem Einschlusskriterium nachselektiert: Es wurden die Patienten bzw. Untersuchungen mit in die finale Auswertung aufgenommen, deren sagittale Schicht im MRT eine maximale Rotationsabweichung von $\pm 3^{\circ}$ von der Neutralposition aufwies.

In Folge dessen wurden 127 Kernspinntomographien von Patienten ausgeschlossen. Die Probanden, insgesamt 56, die den Kriterien entsprachen, wurden ausgewertet (Tabelle 4).

\begin{tabular}{|c|c|c|c|c|}
\hline Geschlecht & $\varnothing$ Alter in Jahren & max Alter & min Alter & Anzahl \\
\hline Männlich & 22,96 & 59 & 13 & 28 \\
\hline Weiblich & 25,64 & 44 & 14 & 28 \\
\hline Gesamt & 24,3 & 59 & 13 & 56 \\
\hline Knieseite & Gesamt & männliche Patienten & weibliche Patienten & \\
\hline Rechts & 28 & 11 & 17 & \\
\hline Links & 28 & 17 & 11 & \\
\hline Gesamt & 56 & 28 & 28 & \\
\hline
\end{tabular}

Tabelle 4: Patientenkollektiv (finale Auswertung, $n=56$ ) 
Das laterale posteriore Kondylen Offset betrug im Mittel 29,64 ( \pm 4,09) mm (min. 14,3 bis max. 39,1 mm), und das mediale posteriore Kondylen Offset betrug im Mittel 34 $( \pm 3,71) \mathrm{mm}(\min .26,3$ bis $\max .44,7 \mathrm{~mm})$ (Tabelle 5).

Die posteriore Kondylenachse $\left(0,11^{\circ} \pm 2,4^{\circ}\right)$ wich nicht signifikant von der funktionellen Flexionsachse ab $(p=0,599)$. Hingegen wichen die $\operatorname{aTEA}\left(4,59^{\circ} \pm 2,47^{\circ}\right)$ und die sTEA $\left(-1,45^{\circ} \pm 2,71^{\circ}\right)$, signifikant von der funktionellen Flexionsachse ab $(p<0,05)$ (Tabelle 5).

\begin{tabular}{|l|r|r|r|r|r||}
\hline & Mittelwert Median Minimum Maximum SD \\
\hline Laterales Offstet in mm & 29,64 & 29,85 & 14,3 & 39,1 & $\pm 4,09$ \\
Mediales Offset in mm & 34 & 33,7 & 26,3 & 44,7 & $\pm 3,71$ \\
Ratio (medial/lateral) & 1,16 & 1,14 & 0,93 & 1,85 & $\pm 0,14$ \\
Abweichung aTEA von fFa in Grad & 4,59 & 4,35 & -1 & 9,8 & $\pm 2,47$ \\
Abweichung PCA von fFa in Grad & 0,11 & 0,07 & $-4,1$ & 5 & $\pm 2,3$ \\
Abweichung sTEA von fFa in Grad & $-1,45$ & -2 & $-6,5$ & 4,8 & $\pm 2,71$ \\
\hline
\end{tabular}

Tabelle 5: Ergebnisse der Messungen ( $n=56)$

In den Abbildungen 13-15 wird der Einfluss des Kondylen-Offset-Ratios auf die Abweichung der anatomischen Achsen (aTEA, sTEA und PCA) zur funktionellen Flexionsachse dargestellt. Es konnte gezeigt werden, dass die Korrelation der Abweichung der aTEA und der sTEA zur Ratio des Kondylen-Offsets statistisch signifikant ist ( $p$-Wert <0,05) (Abbildung 13 und 15). Dabei tendiert die aTEA mit ansteigender Offset-Ratio zunehmend in eine Außenrotationsabweichung. Die aTEA hingegen tendiert mit abnehmende Offset-Ratio zunehmend in eine Innenrotationsabweichung. Bei der Abweichung der PCA zur funktionellen Flexionsachse konnte kein statistisch signifikanter Einfluss durch die KondylenOffset-Ratio gezeigt werden (Abbildung 14). Damit zeigt die PCA unter Berücksichtigung der unterschiedlichen Kondylen-Offset Ratio`s die beste Übereinstimmung mit der funktionellen Flexionsachse. 


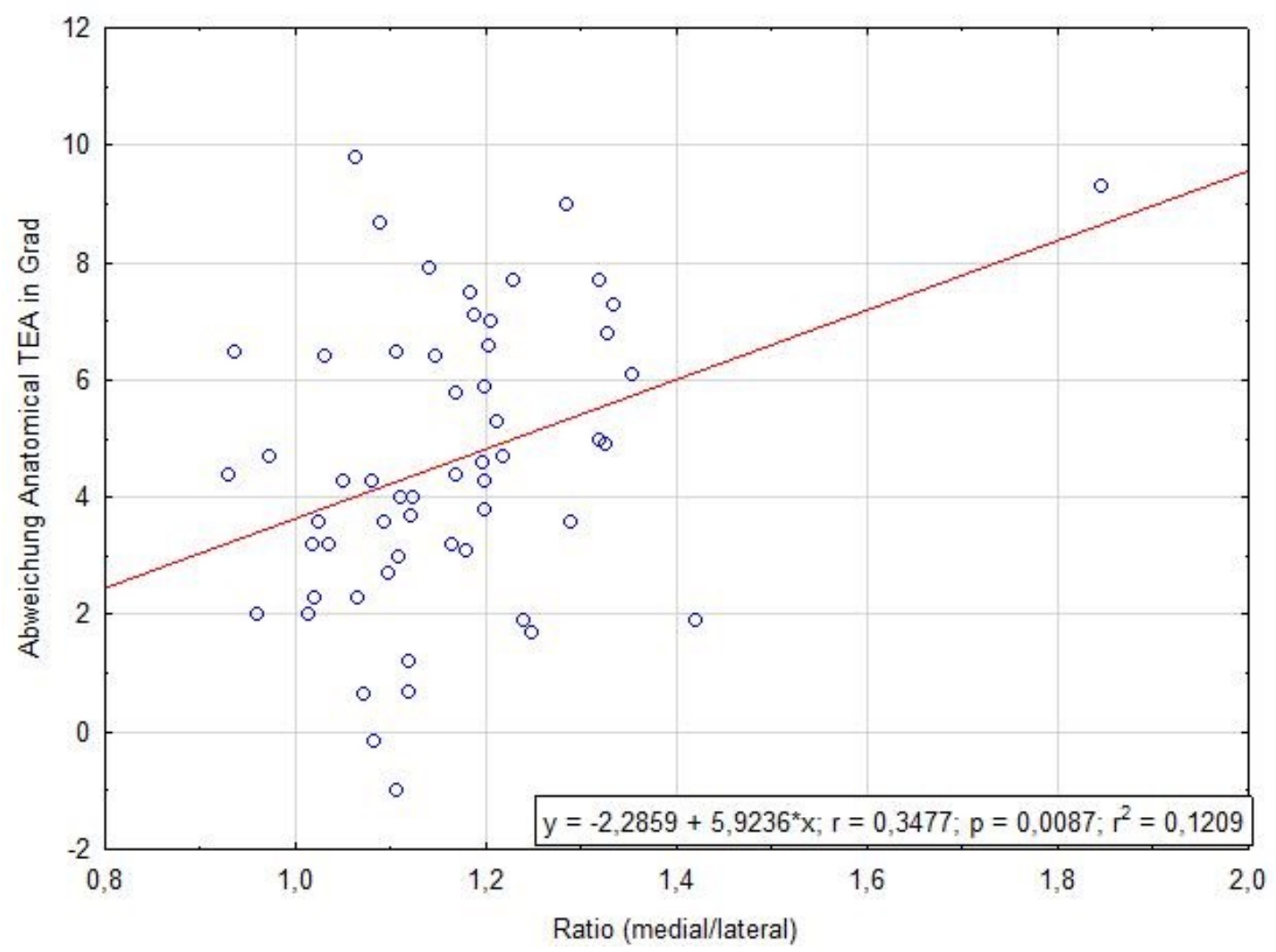

Abbildung 13: Abweichung der anatomischen transepikondylären Achse (aTEA) von der funktionellen Flexionsachse in Grad im Verhältnis zur Ratio des Kondylen Offsets.

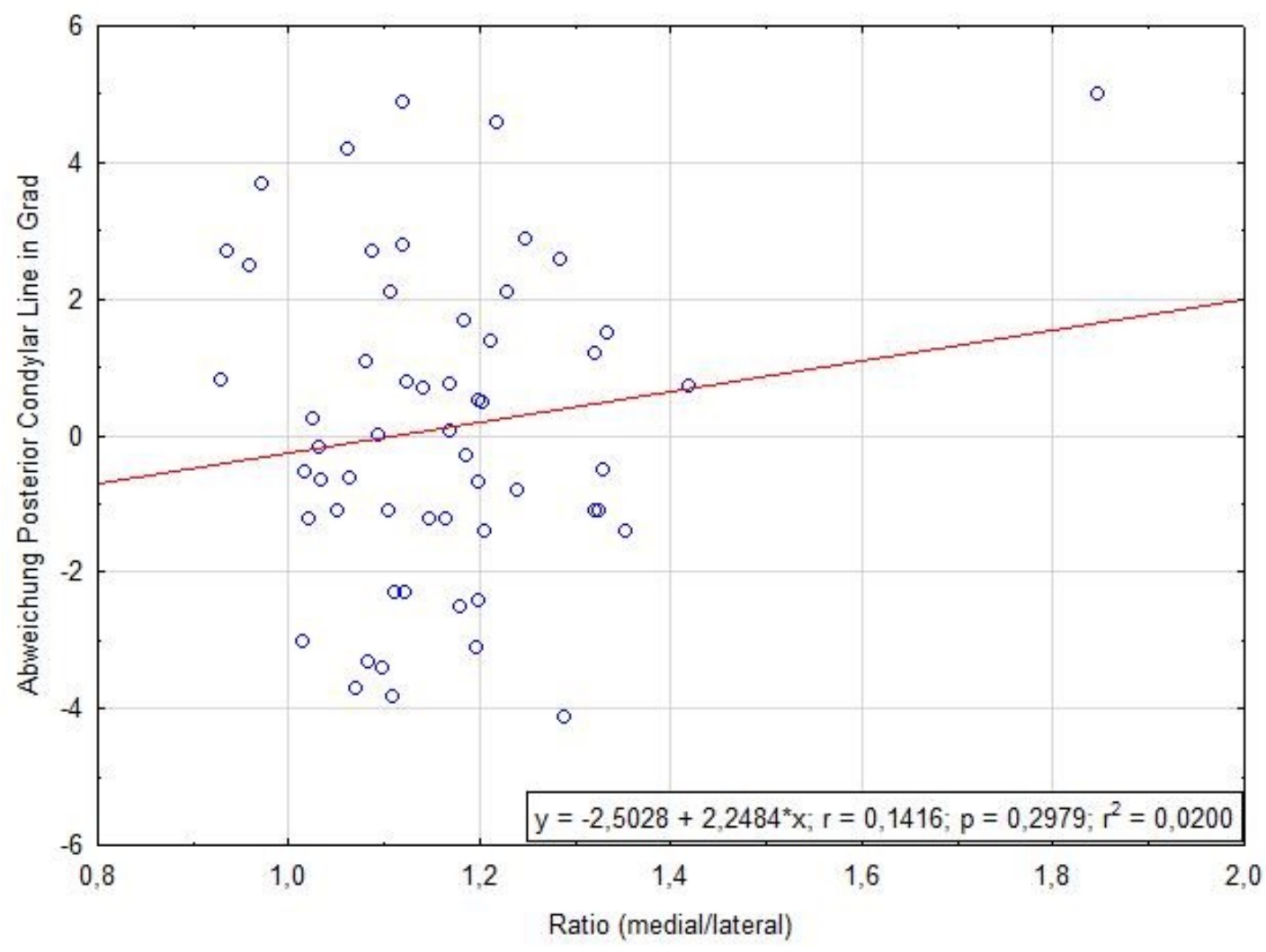

Abbildung 14: Abweichung der posterioren Kondylen Achse (PCA) von der funktionellen Flexionsachse in Grad im Verhältnis zur Ratio des Kondylen Offsets. 


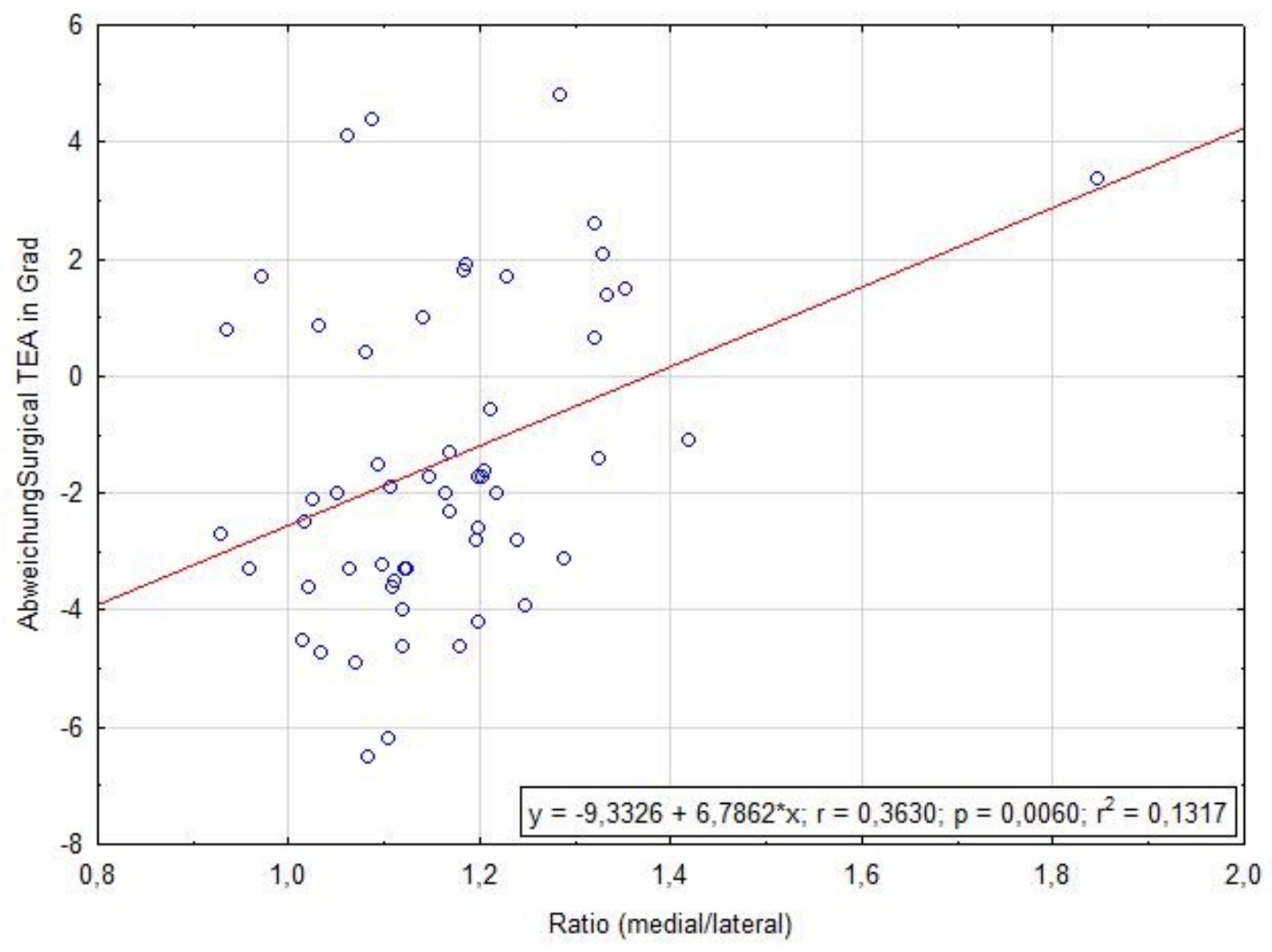

Abbildung 15: Abweichung der chirurgischen transepikondylären Achse (sTEA) von der funktionellen Flexionsachse in Grad im Verhältnis zur Ratio des Kondylen Offsets. 


\section{Diskussion}

\subsection{Hypothese 1}

Es galt zu prüfen ob die Kernspintomographie ein adäquates Verfahren der verlässlichen präoperativen Bestimmung des distalen femoralen Offsets darstellt. In den Messungen ( $n=183$ ) zeigte das laterale Offset eine Streubreite von 14,3 mm bis $41,7 \mathrm{~mm}$, und das mediale Offset eine Streubreite von 21,2 $\mathrm{mm}$ bis 44,7 mm. Die Messungen zeigten ebenfalls, dass die Ratio zwischen dem medialen und dem lateralen Offset in ihrer maximalen Ausdehnung zwischen 0,5 und 1,1 betrug. Hier war bei einer Ratio von 0,5 das laterale Offset um 12,1 mm größer als das mediale Offset, und bei einer Ratio von 1,1 war das mediale Offset 2,8 mm größer als die gegenüberliegende Seite. Bei den Nachmessungen zur Validierung der Intra- und Interreliabilität der Messungen wurden wiederholt die gleichen Werte mit den oben genannten Abweichungen ermittelt. Die beschriebene Messmethode stellt demnach prinzipiell eine verlässliche und reproduzierbare Technik zur Bestimmung des femoralen Offsets dar.

Dennoch wurde in der retrospektiven Analyse die große Differenz des medialen und lateralen Offsets von bis zu 12,1 mm als zu groß empfunden. Bei der Betrachtung der möglichen Fehlerquelle, wurde ein möglicher Rotationsfehler im Rahmen der Untersuchungsplanung bzw. Durchführung in Betracht gezogen. Zu Untersuchungsbeginn wird in der Kernspinntomographie ein Localizer in axialer Schnittrichtung angefertigt. Anhand dieses Localizers werden die sagittalen und die koronaren Untersuchungen geplant. Dieser Vorgang ist nicht standardisiert und birgt mögliche rotatorische Fehlquellen zumal die Messungen des femoralen Offsets an den sagittalen Bildern erfolgt.

Dies führte dazu, dass eine Validitätsstudie durchführt wurde. Es wurde davon ausgegangen, dass eine Rotation im Kniegelenk Einfluss auf die Messungen des femoralen Kondylen-Offsets nach der Cinotti-Methode hat (Kapitel 2.5). Dazu wurden an fünf Probanden insgesamt 30 Messungen durchgeführt. Es zeigte sich, dass eine Innen- / Aussenrotation im Kniegelenk von $5^{\circ}$ einen statistisch signifikanten Einfluss auf die Länge des femoralen Kondylen-Offest medial und lateral hat.

An dem Probandenkollektiv der Validitätsstudie konnte erstmals gezeigt werden, dass eine Außen-/oder Innenrotation des zu untersuchenden Kniegelenks zu einer statistisch signifikanten Änderung des Messergebnisses in der Bestimmung des PCO 
führt. Dies ist gleich zu setzen mit einer rotatorischen Veränderung des Localizers im Rahmen der Planung der Kernspinntomographie.

Mit dem Wissen aus der Validitätsstudie wurde das ursprüngliche Patientenkollektiv erneut gesichtet und nachselektiert. Patienten bei denen die sagittale Schicht nicht $90^{\circ} \pm 3^{\circ}$ zur aTEA als rotatorische Referenzachse ausgerichtet ist wurden um den Rotationseinfluss zu minimieren ausselektiert. Aus den ursprünglich 186 Patienten wurden 56 in die finale Auswertung übernommen. Das laterale posteriore KondylenOffset betrug im Mittel 29,64 ( \pm 4,09) mm (min. 14,3 bis max. 39,1 mm), und das mediale posteriore Kondylen-Offset betrug im Mittel 34 ( \pm 3,71) mm (min. 26,3 bis max. 44,7 mm) (Tabelle 5). Im Vergleich zur ersten Untersuchung zeigte sich eine geringere Streubreite. Dennoch ist die Streubreite auch in dem reduzierten Kollektiv nicht zu vernachlässigen.

Wenn man den rotatorischen Einfluss während der Planung und Durchführung der Untersuchung minimiert hat, stellt die Cinotti Technik eine verlässliche Methode in der MR-Bildgebung zur Bestimmung des posterioren femoralen Kondylen-Offsetts dar.

In der Kniegelenksendoprothetik ist die Kenntnis und die operative Rekonstruktion des posterioren Kondylen-Offset von großer Bedeutung. Es konnte gezeigt werden, dass eine Reduktion des posterioren Kondylen-Offsets um 2 mm eine mögliche Reduktion der postoperativen Beugefähigkeit von $12^{\circ}$ nach Prothesenimplantation bedingen kann (Bellmanns 2005). Dies ist insofern von Bedeutung da die Gelenkfunktion direkt mit der Patientenzufriedenheit korreliert (Bourne 2010).

\subsection{Hypothese 2}

Eine weitere Hypothese war, dass die Größe bzw. die Differenz des medialen und lateralen femoralen Offsets individuell ist und eine große Streubreite zeigt. In den ersten Messungen an 183 Patienten zeigte das laterale Offset eine Streubreite von $14,3 \mathrm{~mm}$ bis $41,7 \mathrm{~mm}$, und das mediale Offsett eine Streubreite von 21,2 $\mathrm{mm}$ bis 44,7 mm. Nach der Validitätsstudie und der Nachselektion wurden 56 Patienten final ausgewertet. Hier zeigte sich eine Größe des lateralen posterioren Kondylen-Offset im Mittel von 29,64 ( \pm 4,09) $\mathrm{mm}$ mit einer Streubreite von min. 14,3 mm bis max. 39,1 $\mathrm{mm}$. Das mediale posteriore Kondylen-Offset betrug im Mittel $34( \pm 3,71) \mathrm{mm}$ mit 
einer Streubreite von min. 26,3 bis max. 44,7 mm (Tabelle 5). Die Streubreite war geringer im Vergleich zum ersten Kollektiv, lag aber weiterhin vor.

Die Beugefähigkeit nach Kniegelenkersatz gilt schon immer als einer der wichtigsten Faktoren der einen Einfluss auf die postoperative Patientenzufriedenheit hat, da viele Aktivitäten des täglichen Lebens abhängig davon sind (Bourne 2010). Für alltägliche Verrichtungen wie das Treppensteigen und das Fahrradfahren benötig der Patient eine Flexion von bis zu $120^{\circ}$ (Rowe 2000; Mulholland 2001). Jedoch haben viele Patienten postoperativ keine Knieflexion von mehr als $90^{\circ}$. Hier kann die fehlerhafte Rekonstruktion des femoralen Offset neben anderen möglichen Faktoren die schlechte Beugefähigkeit bedingen. Dabei wird bei reduziertem Offset ein frühzeitiges Anschlagen des Femurs an die dorsale Tibia mit entsprechendem Impingement als Ursache der Beugehemmung beschrieben. Eine Verkürzung des posterioren femoralen Kondylen-Offsetts um $3 \mathrm{~mm}$ nach Kniegelenkstotalendoprothese, kann die Kniegelenksflexion um $10^{\circ}$ reduzieren bis es zu einem tibio-femoralen Impingement kommt (Massin 2003).

Zusätzlich kann durch eine übermäßige Verkürzung des femoralen Offsets ein größerer Flexionsraum im Kniegelenk mit erhöhter Flexionsinstablität und letztendlich möglichen pathologischen Abgleiten des Femurs nach vorne entstehen (Bellemans 2009).

Aufgrund des oben dargestellten sollte zur Vermeidung dieser Probleme das individuelle posteriore Kondylen-Offset des Patienten präoperativ bekannt sein und eine möglichst exakte Wiederherstellung intraoperativ erfolgen.

\subsection{Hypothese 3}

In der Kniegelenksendoprothetik erfolgt in den meisten Fällen die rotatorische Ausrichtung der femoralen Komponente unter Anwendung sogenannter anatomischer Landmarken. So galt als weitere Hypothese, dass die in der Kniegelenksendoprothetik beschriebenen Rotationsachsen (aTEA, sTEA und PCA) nur ungenügend mit der individuellen funktionellen Flexionsachse korrelieren.

Die funktionelle Flexionsachse liegt im distalen Femur und verläuft jeweils durch die Mitte der medialen und der lateralen Femurkondyle (Hollister 1993; Stiehl 1995). Sie 
gilt als die Achse in der das gesunde menschliche Kniegelenk zwischen 30-90 Flexion beugt. Im Rahmen der totalen Knieendoprothetik gilt diese funktionelle Flexionsachse als Goldstandart, die es als rotatorische Achse zu rekonstruieren gilt. Die funktionelle Flexionsachse lässt sich allerdings intraoperativ nicht ermitteln. Aus diesem Grund verwenden die Operateure die beschriebenen anatomischen Landmarken zur intraoperativen femoralen Rotationsausrichtung.

In den Untersuchungen der 56 Patienten zeigte die aTEA eine Abweichung von der funktionellen Flexionsachse im Mittelwert von 4,59 ${ }^{\circ}$, der Median lag bei 4,35 ${ }^{\circ}$ (Min.: $1^{\circ}$; Max.: $9,8^{\circ}$ ) und einer Standardabweichung von $\pm 2,47^{\circ}$. Die PCA zeigte eine Abweichung von der funktionellen Flexionsachse im Mittelwert von $0,11^{\circ}$, der Median lag bei $0,07^{\circ}$ (Min.: $-4,1^{\circ}$; Max.: $5^{\circ}$ ) und einer Standardabweichung von $\pm 2,3^{\circ}$. Die STEA hatte eine Abweichung von der funktionellen Flexionsachse im Mittelwert von 1,45 $5^{\circ}$, der Median lag bei $-2^{\circ}$ (Min.: $-6,5^{\circ}$; Max.: 4, $8^{\circ}$ ) und einer Standardabweichung von $\pm 2,71^{\circ}$

Es zeigte sich die PCA als diejenige, die die geringste Abweichung zur funktionellen Flexionsachse aufweist. Alle Achsen besaßen annähernd die gleiche Standartabweichung.

In der bereits 2010 publizierten Studie von Walde et al. wurde zur intraoperative Bestimmung der femoralen Rotation eine bandspannungsadaptierte Technik verwendet (Walde 2010). Es konnte ebenfalls gezeigt werden, dass die anatomischen Achsen einer hohen Variabilität unterliegen und deren intraoperative Detektion eher der Erfahrung des Operateurs unterliegt (Siston 2005). Im Gegenzug dazu konnte die von Walde et al. beschriebene bandspannungsadaptierte Technik eine reproduzierbare funktionale femorale Rotation mit der Erzeugung eines ausbalancierten Beugespaltes erzielen (Walde 2010).

Die Erzeugung eines parallelen bandbalancierten Beugespalts gilt als eine der Voraussetzungen für eine gute Gelenkfunktion nach Kniegelenkersatz (Walde 2010). Eine fehlerhafte rotatorische Ausrichtung der femoralen Komponente kann zu einer schmerzhaft reduzierten postoperativen Beugefähigkeit führen (Hofmann 2003). Dies ist unter anderem in einer resultierenden erhöhten Bandspannung mit kontralateraler Instabilität begründet. Zusätzlich kann eine fehlerhafte femorale Rotation zu einem 
schmerzhafte Patella maltracking bis hin zu einer patello-femoralen Instabilität führen (Hofmann 2003)

Die hier dargestellten Ergebnisse zeigen, dass eine reliable rotatorische Ausrichtung der femoralen Komponenten mit den anatomischen Landmarken nur eingeschränkt durchführbar ist. Alternative Operationstechniken zur funktionellen femoralen Rotationsausrichtung existieren. Diese haben aber noch keinen Einzug in die Standardversorgung der Patienten mit Kniegelenkersatz gefunden (Walde 2010).

\subsection{Hypothese 4}

In den letzten Jahren wurden kontroverse Ergebnisse über den Einfluss und die Verwendung der anatomischen Landmarken zur femoralen Rotationsausrichtung in der Knieendoprothetik veröffentlicht (Massin 2006; Bellemans 2005). Allerdings wurde in keiner Veröffentlichung bisher der Einfluss des femoralen posterioren Kondylen-Offsets und einer Abweichung der bekannten Landmarken zur fFA beschrieben. In dieser Untersuchung wurde das Femurkondylen-Offset und die Abweichung der aTEA, sTEA und PCA von der fFA mit Hilfe von Kernspintomographien ermittelt. Die Messungen wurden an der lateralen und der medialen Kondyle getrennt durchgeführt um auch Unterschiede identifizieren zu können, die man in einer konventionellen Röntgenaufnahme nicht erkennen kann (Hudek 2009).

So wurde als weitere Hypothese angenommen, dass die unterschiedlichen Größen des medialen und lateralen Kondylen-Offsets, einen Einfluss auf die Genauigkeit der anatomischen Achsen zur femoralen Rotationsausrichtung in der Kniegelenksendoprothetik haben (PCA, aTEA und STEA). In dieser Studie konnte diese Hypothese bestätigt werden. Die Größe des posterioren FemurkondylenOffsets innerhalb des Knies, beziehungsweise deren Ratio zueinander haben signifikanten Einfluss auf die Abweichung der aTEA und STEA zur funktionellen Flexionsachse $(p<0,05)$, d.h. positiv signifikant korreliert nur die aTEA und die sTEA zum Kondylen-Offset Ratio.

Hierbei konnten in dieser Arbeit erstmals folgende Ergebnisse genau beschrieben werden: Die aTEA führt bei einem ansteigenden Offset-ratio zu einer zunehmenden Außenrotation während PCA und sTEA bei einer Offset-ratio <1 eher zu einer Innenrotationsabweichung führen. 
Die Ergebnisse zeigten auch, dass die größte Zuverlässigkeit, bezüglich des femoralen Offsets und die geringste Abweichung zur fFA, die PCA zeigt ( $p=0,298 \mathrm{~s}$. Abbildung 14).

Aus der Studie wären folgende Inhalte potentiell als Konsequenz in den klinischen Allag übertragbar: Vor der geplanten Implantation einer Kniegelenkstotalendoprothese ist eine sorgfältige Interpretation der Bildgebung notwendig und man sollte sich der möglichen Fehlerquelle einer Fehlrotation in der Kernspinntomographie bewusst sein. Die bisher bekannten konventionellen Achsen sind keine idealen intraoperativen Referenzpunkte. Dies wird darüber hinaus erschwert, dass die eigentliche funktionelle Flexionsachse intraoperativ nicht bestimmt werden kann. Da die PCA am wenigsten von der fFA abweicht, kann die PCA als mögliche anatomische Landmarke intraoperativ verwendet werden. Man sollte in Betracht ziehen, dass es hilfreich für einen Operateur ist, den möglichst genauen Verlauf der funktionellen Flexionsachse im Knie seines Patienten zu kennen. Hierbei reicht es oftmals jedoch nicht aus, erst während der Operation sich an anatomischen Referenzpunkten zu orientieren. Vielmehr wäre zur Optimierung der prothetischen Versorgung ratsam eine präoperative Bildgebung mittels MRT, Computertomographie (CT) oder im Idealfall sogar eine dreidimensionale Rekonstruktion durch die gewonnen Bilder zur besseren und gezielteren präoperativen Planung, durchzuführen. Hierbei kann dann im Vorhinein eine gezielte Achsenkorrektur der Sägeschnittführung bei der Präparation des distalen Femurs geplant werden. Auf diese Weise wäre es möglich, Operationsergebnisse zu verbessern und nachfolgende Komplikationen und Fehlstellungen zu vermeiden.

Die Studie hat aber auch ihre Grenzen. So wurde das femorale Offset in einer transversalen Schnittebene gemessen, während die Achsen und deren Abweichungen von der fFA in einem anderen, horizontalen MRT-Schnitt gemessen wurden. Um mögliche Übertragungsfehler auszuschließen wurden alle Patienten mehrfach in einem zeitlichen Abstand gemessen. Auch wurden in den verwendeten MRT-Bildern keine extra dünnen Schichtaufnahmen gefahren, sodass nicht jedes Knie lückenlos rekonstruiert werden konnte. Das posteriore Kondylen-Offset wurde als eine gerade Linie die im rechten Winkel von der verschobenen Femurachse auf die am weitesten posterior gelegene Stelle der Femurkondyle gemessen. Jedoch wurden nur Knie von nicht-arthrotischen Patienten gemessen und die Ergebnisse 
wären vielleicht anders bei arthrotischen veränderten Knien ausgefallen. Die Studie ist auch keine dynamische Echtzeitstudie, bei den MRT Aufnahmen waren die Patienten im MRT mit gestrecktem Bein fixiert.

Als Fazit lässt sich sagen, dass das Wissen regelrechter anatomischer Verhältnisse im Kniegelenk die nötige Voraussetzung bildet, um die Rolle der einzelnen Knochenund Gewebebestandteile im Kniegelenk zu verstehen, die eine balancierte Flexion ermöglichen. Die Studie hat gezeigt, dass die Ratio des posterioren Kondylen-Offsets mit einer Abweichung der etablierten anatomischen Femurachsen zur funktionellen Flexionsachse einhergeht. Als zuverlässigste Achse zeigte sich die PCA. Bei der STEA und aTEA kann es zu Fehlinterpretationen sowohl in der Innen- als auch in der Außenrotation kommen. 


\section{Zusammenfassung}

Die Erzeugung eines parallelen, balancierten Beugespalts gilt als eine der Voraussetzungen in der Kniegelenksendoprothetik. Die intraoperative Bestimmung der korrekten femoralen Rotation hat daher eine große Bedeutung für den Erfolg der Operation. Bei der Referenzierung an den bekannten anatomischen Landmarken (PCA, aTEA, STEA) erhält man postoperativ unterschiedliche Rotationsergebnisse der femoralen Prothesenkomponente. Es stellte sich daher die Frage, ob die unbefriedigenden Ergebnisse einiger Patienten nach Implantation einer KnieTotalendoprothese möglicherweise auf eine Abweichung der femoralen Rotation von der funktionellen Flexionsachse zurückgeführt werden können.

Neben der femoralen Rotation stellt die intraoperative Rekonstruktion des posterioren Kondylen-Offsets eine weitere wichtige Variable für den Operationserfolg in der Knieendoprothetik dar. Daher ist eine exakte präoperative Bestimmung des posterioren Kondylen-Offsets bei jedem Patienten notwendig.

Es wurden vier Hypothesen vor Studienbeginn definiert:

1. Die Kernspintomographie stellt ein adäquates Verfahren zur verlässlichen präoperativen Bestimmung des posterioren femoralen Offsets dar.

2. Die Größe bzw. die Differenz des medialen und lateralen femoralen Offsets ist individuell und zeigt eine große Streubreite.

3. Die in der Kniegelenksendoprothetik beschriebenen Rotationsachsen (aTEA, sTEA und PCA) korrelieren nur ungenügend mit der individuellen funktionellen Flexionsachse.

4. Das posteriore Kondylen-Offset hat einen statistisch signifikanten

Einfluss auf die Genauigkeit der anatomischen Rotationsachsen (aTEA, sTEA und PCA) zur funktionellen Flexionsachse

Bei der Evaluation der ersten Ergebnisse stellte die Kernspintomographie ein adäquates Verfahren zur Bestimmung des PCO dar. Es zeigte sich jedoch eine große Streubreite der Messungen die einer weiteren Validierung bedurfte. Es wurde eine Validitätsstudie durchgeführt. Die folgende Auswertung zeigte, dass bei einer 
Innen- oder Außenrotation des Kniegelenkes es zu Fehlmessungen des PCO kommt. Empfehlungen zur korrekten Durchführung bzw. Auswertung von Kernspintomographien konnten aufgestellt werden. Nach Kenntnis des rotationsbedingten Fehlers wurden die Patienten nachselektiert $(n=56)$. Die Streubreite war nun deutlich geringer lag aber weiterhin vor. Trotz Vermeidung des Rotationseinflusses finden sich individuelle Unterschiede in der Länge des PCO.

In der Kniegelenksendoprothetik ist die Rekonstruktion der funktionellen Flexionsachse der zu erreichende Goldstandart, kann aber intraoperativ nicht bestimmt werden, daher bedient man sich intraoperativ anatomischen Landmarken. Es konnte gezeigt werden, dass von den anatomischen Landmarken die PCA am ehesten mit der fFA korreliert und von den Landmarken am ehesten empfohlen werden kann.

Die Ergebnisse dieser Studie zeigen weiter, dass die Genauigkeit der bis dato verwendeten anatomischen Landmarken (aTEA, STEA, PCA ) zur möglichst optimalen Wiederherstellung der individuellen fFA von Kondylen-Offset beeinflusst werden. Dabei kann es in Abhängigkeit des Offset-Ratios zur vermehrten Innen- als auch Außenrotationsabweichungen kommen. Die PCA bietet hierbei die verlässlichste Größe. Für den klinischen Altag in der Kniegelenksendoprothetik heißt dies, dass die Kenntnis der individuellen Streubreite des PCO nicht nur in die Planung der femoralen Rotation mit einbezogen werden sollte, sondern sollte ebenfalls Einfluss auf die gewählte OP-Technik haben. Hier lassen sich vielleicht in Zukunft durch exaktere Planung postoperative Komplikationen reduzieren und die Patientenzufriedenheit steigern. 


\section{Abbildungsverzeichnis}

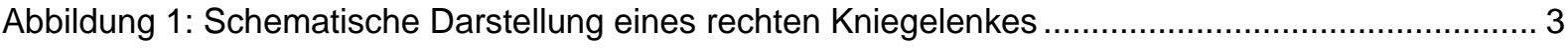

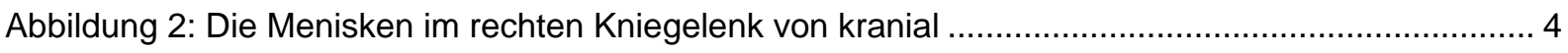

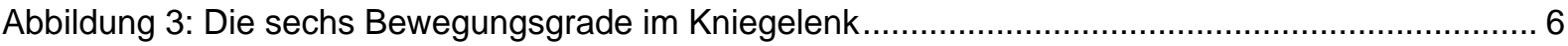

Abbildung 4: Schematischer Sagittalschnitt durch eine Femurkondyle ........................................... 10

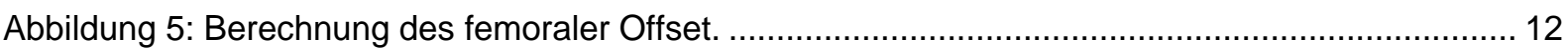

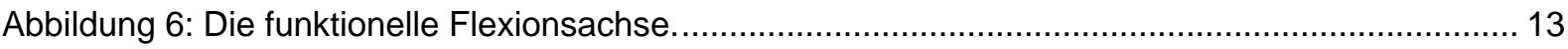

Abbildung 7: Femur in axialer Ebene, mit den Referenzachsen in der Kniegelenksendoprothetik ...... 15

Abbildung 8: Ausrichten des Lokalizers an einer axialen Schicht im MRT ........................................ 20

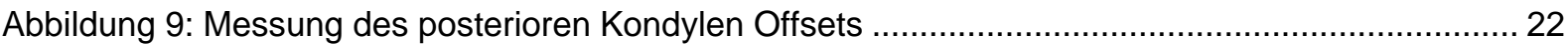

Abbildung 10: Berechnung der Rotationsachse in der posterioren Femurkondyle.............................. 23

Abbildung 11: fFA im Verhältnis zu den konventionellen Achsen................................................. 24

Abbildung 12: Abweichung der PCO-Länge bei unterschiedlichen Rotationsstellungen des Knies. .. 28

Abbildung 13: Abweichung der aTEA von der fFA im Verhältnis zur Ratio des Kondyle Offsets......... 31

Abbildung 14: Abweichung der PCA von der fFA im Verhältnis zur Ratio des Kondyle Offsets........... 31

Abbildung 15: Abweichung der sTEA von der fFA im Verhältnis zur Ratio des Kondyle Offsets. ........ 32 


\section{$7 \quad$ Tabellenverzeichnis}

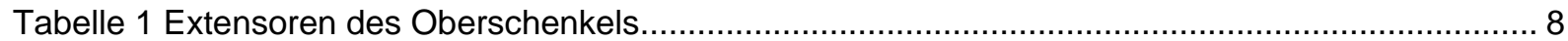

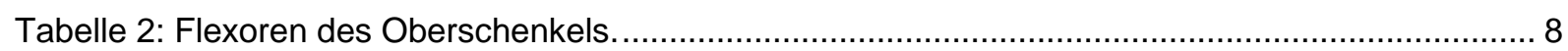

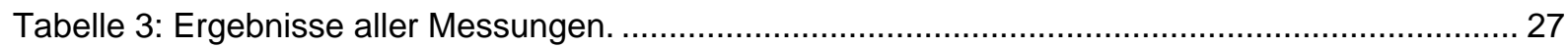

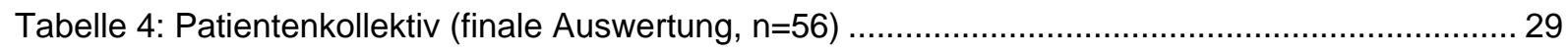

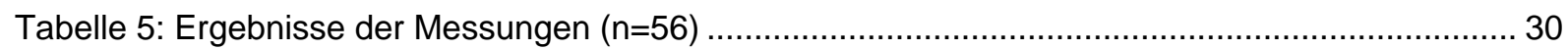




\section{$8 \quad$ Literaturverzeichnis}

Adachi N, Ochi M, Uchio Y, Iwasa J, Ryoke K, Kuriwaka M (2002): Mechanoreceptors in the anterior cruciate ligament contribute to the joint position sense. Acta Orthop Scand 73 , 330-334

Akagi M, Matsusue Y, Mata T, Asada Y, Horiguchi M, Iida H, Nakamura T (1999): Effect of rotational alignment on patellar tracking in total knee arthroplasty. Clin Orthop Relat Res $\underline{14}$, 155-163

Amis AA, Dawkins GP (1991): Functional anatomy of the anterior cruciate ligament. Fibre bundle actions related to ligament replacements and injuries. J Bone Joint Surg Br 73, 260267

Andriacchi TP, Stanwyck TS, Galante JO (1986): Knee biomechanics and total knee replacement. J Arthroplasty $\underline{1}$, 211-219

Asano T, Akagi M, Nakamura T (2005): The functional flexion-extension axis of the knee corresponds to the surgical epicondylar axis: in vivo analysis using a biplanar image-matching technique. J Arthroplasty 20, 1060-1067

Balcarek P, Terwey A, Jung K, Walde TA, Frosch S, Schüttrumpf JP, Wachowski MM, Dathe H, Stürmer KM (2013): Influence of tibial slope asymmetry on femoral rotation in patients with lateral patellar instability. Knee Surg Sports Traumatol Arthrosc 21, 2155-2163

Bellemans J, Robijns F, Duerinckx J, Banks S, Vandenneucker H (2005): The influence of tibial slope on maximal flexion after total knee arthroplasty. Knee Surg Sports Traumatol Arthrosc 13, 193-196

Berger RA, Rubash HE, Seel MJ, Thompson WH, Crossett LS (1992): Determining the rotational aligment of the femoral component in total knee arthroplasty using the epicondylar axis. Clin Orthop Relat Res $\underline{9}, 18-24$

Biscević M, Hebibović M, Smrke D (2005): Variations of femoral condyle shape. Coll Antropol 29, 409-414

Bourne RB, Chesworth BM, Davis AM, Mahomed NN, Charron K (2010): Patient satisfaction after total knee arthroplasty: Who is satisfied and who is not? Clin Orthop Relat Res 1, 57-63

Cinotti G, Sessa P, Ripani FR, Postacchini R, Masciangelo R, Giannicola G (2012):

Correlation between posterior offset of femoral condyles and sagittal slope of the tibial plateau. Anat 221, 452-458

Dargel J, Schmidt-Wiethoff R, Feiser J, Koebke J, Schlüter-Brust K, Eysel P, Michael JW (2011): Relationship between human femorotibial joint configuration and the morphometry of the anterior cruciate ligament. Arch Orthop Trauma Surg 131, 1095-1105

Essinger JR, Leyvraz PF, Heegard JH, Robertson DD (1989): A mathematical model for the evaluation of the behaviour during flexion of condylar-type knee prostheses. J Biomech 22 , 1229-1241 
Frankel VH, Burstein AH, Brooks DB (1971): Biomechanics of internal derangement of the knee. Pathomechanics as determined by analysis of the instant centers of motion. J Bone Joint Surg Am 53, 945-962

Freeman M, Pinskerova V (2005): The movement of the normal tibio-femoral joint. J Biomech 38, 197-208

Fu FH, Harner CD, Johnson DL, Miller MD, Woo SL (1994): Biomechanics of knee ligaments: basic concepts and clinical application. Instr Course Lect $\underline{43}$, 137-148

Furman W, Marshall JL, Girgis FG (1976): The anterior cruciate ligament. A functional analysis based on postmortem studies. J Bone Joint Surg Am 모, 179-185

Goodfellow J, O'Connor J (1978): The mechanics of the knee and prosthesis design. J Bone Joint Surg Br 60, 358-369

Griffin FM, Insall JN, Scuderi GR (2000): Accuracy of soft tissue balancing in total knee arthroplasty. J Arthroplasty $\underline{15}$, 970-973

Hanson GR, Suggs JF, Freiberg AA, Durbhakula S, Li G (2006): Investigation of in vivo 6DOF total knee arthoplasty kinematics using a dual orthogonal fluoroscopic system. J Orthop Res 24, 974-981

Hofmann S, Romero J, Roth-Schiffl E, Albrecht T (2003): Rotationsfehlstellungen der Komponenten als Ursache chronischer Schmerzen und vorzeitigem Prothesenversagen bei Knieendoprothesen. Orthopade 32, 469-476

Hollister AM, Jatana S, Singh AK, Sullivan WW, Lupichuk AG (1993): The axes of rotation of the knee. Clin Orthop Relat Res $\underline{13}$, 259-268

Hudek R, Schmutz S, Regenfelder F, Fuchs B, Koch PP (2009): Novel measurement technique of the tibial slope on conventional MRI. Clin Orthop Relat Res 467, 2066-2072 Hughston JC, Bowden JA, Andrews JR, Norwood LA (1980): Acute tears of the posterior cruciate ligament. Results of operative treatment. J Bone Joint Surg Am 62, 438-450

Igbigbi PS, Sharrif M (2005): The bicondylar angle of adult Malawians. Am J Orthop $\underline{34}$, 291-294

Iwaki H (2000): Tibiofemoral movement: the shapes and relative movements of the femur and tibia in the unloaded cadaver knee. J Bone Joint Surg Br 2000, 1189-1195

Kettelkamp DB, Johnson RJ, Smidt GL, Chao EY, Walker M (1970): An electrogoniometric study of knee motion in normal gait. J Bone Joint Surg Am 52, 775-790

Laskin RS (1995): Flexion space configuration in total knee arthroplasty. J Arthroplasty $\underline{10}$, 657-660

Laskin RS, Riegèr MA (1989): The surgical technique for performing a total knee replacement arthroplasty. Orthop Clin North Am 20, 31-48

Laubenthal KN, Smidt GL, Kettelkamp DB (1972): A quantitative analysis of knee motion during activities of daily living. Phys Ther $\underline{52}$, 34-43

Lotke PA, Ecker ML (1977): Influence of positioning of prosthesis in total knee replacement. J Bone Joint Surg Am 모, 77-79 
Mantas JP, Bloebaum RD, Skedros JG, Hofmann AA (1992): Implications of reference axes used for rotational alignment of the femoral component in primary and revision knee arthroplasty. J Arthroplasty $\underline{7}$, 531-535

Massin P, Gournay A (2006): Optimization of the posterior condylar offset, tibial slope, and condylar roll-back in total knee arthroplasty. J Arthroplasty 21, 889-896

Matsuda S, Matsuda H, Miyagi T, Sasaki K, Iwamoto Y, Miura H (1998): Femoral condyle geometry in the normal and varus knee. Clin Orthop Relat Res $\underline{123}$, 183-188

Miller MC, Berger RA, Petrella AJ, Karmas A, Rubash HE (2001): Optimizing femoral component rotation in total knee arthroplasty. Clin Orthop Relat Res $\underline{304}$, 38-45

Mulhall KJ, Ghomrawi HM, Scully S, Callaghan JJ, Saleh KJ (2006): Current etiologies and modes of failure in total knee arthroplasty revision. Clin Orthop Relat Res $\underline{446}$, 45-50

Mulholland SJ, Wyss UP (2001): Activities of daily living in non-Western cultures: range of motion requirements for hip and knee joint implants. Int J Rehabil Res 24(3), 191-2008

Olcott CW, Scott RD (1999): The Ranawat Award. Femoral component rotation during total knee arthroplasty. Clin Orthop Relat Res 215, 39-42

Pinskerova V, Iwaki H, Freeman MA (2000): The shapes and relative movements of the femur and tibia at the knee. Orthopade 29, 3-5

Platzer W: Bewegungsapparat (Taschenatlas der Anatomie in 3 Bänden). Band 1; 10.

Auflage; Thieme, Stuttgart 2009

Putz R, Mühlhofer H, Ercan Y (2007): Bänder des Kniegelenks. Orthopade 36, 612, 614-9

Renström P, Johnson RJ (1990): Anatomy and biomechanics of the menisci. Clin Sports Med 9, 523-538

Ritter MA, Campbell ED (1987): Effect of range of motion on the success of a total knee arthroplasty. J of Arthrop 2, $95-97$

Ritter MA, Lutgring JD, Davis KE, Berend ME (2008): The effect of postoperative range of motion on functional activities after posterior cruciate-retaining total knee arthroplasty. $\mathrm{J}$ Bone Joint Surg Am 90, 777-784

Rowe PJ, Myles CM, Walker C, Nutton R (2000): Knee joint kinematics in gait and other functional activities measured using flexible electrogoniometry: how much knee motion is sufficient for normal daily life? Gait Posture 12(2), 143-155

Santavirta S, Konttinen Y, Lappalainen R, Anttila A, Goodman S, Lind M, Smith L, Takagi M, Gómez-Barrena E, Nordsletten L (1998): Materials in total joint replacement. Curr Ortho $\underline{12}, 51-57$

Schiebler TH, Korf HW: Anatomie: Histologie, Entwicklungsgeschichte, makroskopische und mikroskopische Anatomie, Topographie - unter Berücksichtigung des Gegenstandskatalogs. 10. Auflage; Steinkopff, Darmstadt 2007

Schroeder-Boersch H (2001): Gelenkmechanik und das Design moderner Knieprothesen-Zeit zum Umdenken! Z Orthop Ihre Grenzgeb $\underline{139}$, 3-7 
Schünke M, Schulte E, Schumacher U, Rude J, Voll M, Wesker K: Allgemeine Anatomie und Bewegungssystem (Prometheus Lern Atlas der Anatomie in 3 Bänden). Band 1; Thieme, Stuttgart 2005

Shefelbine SJ, Tardieu C, Carter DR (2002): Development of the femoral bicondylar angle in hominid bipedalism. Bone $\underline{30}$, 765-770

Siston RA, Patel JJ, Goodman SB, Delp SL, Giori NJ (2005): The variability of femoral rotational alignment in total knee arthroplasty. J Bone Joint Surg Am 87, 2276-2280

Skolnick MD, Coventry MB, Ilstrup DM (1976): Geometric total knee arthroplasty. A twoyear follow-up study. J Bone Joint Surg Am $\underline{58}$, 749-753

Soudan K, van Audekercke R, Martens M (1979): Methods, difficulties and inaccuracies in the study of human joint kinematics and pathokinematics by the instant axis concept. Example: the knee joint. J Biomech 12, 27-33

Stiehl JB, Abbott BD (1995): Morphology of the transepicondylar axis and its application in primary and revision total knee arthroplasty. J Arthroplasty 10, 785-789

Tardieu C, Damsin JP (1997): Evolution of the angle of obliquity of the femoral diaphysis during growth--correlations. Surg Radiol Anat 19, 91-97

Tardieu C, Glard Y, Garron E, Boulay C, Jouve JL, Dutour O, Boetsch G, Bollini G (2006): Relationship between formation of the femoral bicondylar angle and trochlear shape: Independence of diaphyseal and epiphyseal growth. Am J Phys Anthropol 130, 491-500

Victor J (2009): Rotational alignment of the distal femur: A literature review. Ortho Trauma: Surgery \& Research $\underline{95}$, 365-372

Walde TA, Bussert J, Sehmisch S, Balcarek P, Stürmer KM, Walde HJ, Frosch KH (2010): Optimized functional femoral rotation in navigated total knee arthroplasty considering ligament tension. Knee 17, 381-386

Walker PS, Erkman MJ (1975): The role of the menisci in force transmission across the knee. Clin Orthop Relat Res 7, 184-192

Woo SL, Debski RE, Withrow JD, Janaushek MA (1999): Biomechanics of knee ligaments. Am J Sports Med 27, 533-543

Wülker N (Hrsg.): Taschenlehrbuch Orthopädie und Unfallchirurgie. Thieme, Stuttgart 2009

Yoshioka Y, Siu D, Cooke TD (1987): The anatomy and functional axes of the femur. J Bone Joint Surg Am 으, 873-880

\section{Publikation:}

Frosch S, Brodkorb T, Schüttrumpf JP, Wachowski MM, Walde TA, Stürmer KM, Balcarek P (2014): Characteristics of femorotibial joint geometry in the trochlear dysplastic femur. J Anat 225, 367-373 